\title{
Instantons and some concordance invariants of knots
}

\author{
P. B. Kronheimer and T. S. Mrowka \\ Harvard University, Cambridge MA 02138 \\ Massachusetts Institute of Technology, Cambridge MA 02139
}

\begin{abstract}
Concordance invariants of knots are derived from the instanton homology groups with local coefficients, as introduced in earlier work of the authors. These concordance invariants include a 1-parameter family of homomorphisms $f_{r}$, from the knot concordance group to $\mathbb{R}$. Prima facie, these concordance invariants have the potential to provide independent bounds on the genus and number of double points for immersed surfaces with boundary a given knot.
\end{abstract}

\section{Contents}

1 Introduction 2

2 Review of instanton homology with local coefficients 5

3 The ideal of a knot 9

4 Non-orientable surfaces 16

5 Reduced homology and concordance homomorphisms 22

6 Examples 27

7 Unknotting number and other properties 32

8 Calculation for the trefoil $\quad 35$

9 Further calculations 43

The work of the first author was supported by the National Science Foundation through NSF grants DMS-1405652 and DMS-1707924. The work of the second author was supported by NSF grants DMS-1406348 and DMS-1808794, and by a grant from the Simons Foundation, grant number 503559 TSM. 


\section{Introduction}

\section{Concordance invariants from instanton homology}

For a knot $K$ in a closed, oriented 3-manifold $Y$, the authors' earlier papers have introduced instanton homology groups $I^{\sharp}(Y, K)$, and reduced instanton homology groups $I^{\natural}(K)$. Several variants of the basic construction are possible. In particular, in [9], a version of instanton homology with local coefficients $I^{\sharp}(Y, K ; \Gamma)$ is constructed. Here $\Gamma$ is a local system of coefficients over an appropriate configuration space of connections associated to $(Y, K)$. It is a system of free rank-1 $\mathscr{R}$-modules, where $\mathscr{R}$ is the ring of finite Laurent series in 4 variables over the field of two elements:

$$
\mathscr{R}=\mathbb{F}_{2}\left[T_{0}^{ \pm 1}, T_{1}^{ \pm 1}, T_{2}^{ \pm 1}, T_{3}^{ \pm 1}\right]
$$

Given a base change $\sigma: \mathscr{R} \rightarrow \mathcal{S}$, we write $\Gamma_{\sigma}$ for the local system $\Gamma \otimes_{\sigma} \mathcal{S}$, and we can construct the groups $I^{\sharp}\left(Y, K ; \Gamma_{\sigma}\right)$. If $\sigma$ satisfies the condition $\sigma\left(T_{0}\right)=\sigma\left(T_{1}\right)$, then the reduced groups $I^{\natural}\left(Y, K ; \Gamma_{\sigma}\right)$ are also defined. In the case of the unknot, the reduced group is a free $\mathcal{S}$-module of rank 1 , while the unreduced group is free of rank 2. For details we refer to [9] and the references therein, though we shall summarize some features in Section 2.

The purpose of this paper is to explore how these instanton homology groups with local coefficients give rise to (potentially) new concordance invariants of knots. In its "raw" form, the first such invariant associates to a knot $K \subset S^{3}$ a fractional ideal

$$
z^{\sharp}(K) \subset \operatorname{Frac}(\mathscr{R}) .
$$

There is also a version of this construction using the reduced version $I^{\natural}(K ; \Gamma)$ in place of $I^{\sharp}(K ; \Gamma)$ giving rise to a potentially different ideal,

$$
z_{\sigma}^{\natural}(K) \subset \operatorname{Frac}(\delta) .
$$

for any base-change $\sigma$ with $\sigma\left(T_{0}\right)=\sigma\left(T_{1}\right)$. Other invariants can then be derived from this construction. In particular if we have a base-change $\sigma: \mathscr{R} \rightarrow \mathcal{S}$ where $\delta$ is a valuation ring, and if $\sigma\left(T_{0}\right)=\sigma\left(T_{1}\right)$, then one may construct a homomorphism

$$
f_{\sigma}: \text { Conc } \rightarrow \operatorname{Val}(\delta),
$$

where Conc is the concordance group of knots and $\operatorname{Val}(\mathcal{S})$ is the valuation group of $\mathcal{S}$. (See Section 5 below.) 
Similar constructions, using Heegaard Floer homology rather than instanton homology, have been made earlier by Ozsvath and Szabo in [13] and by Alishahi and Eftekhary in [3]. In the context of gauge theory, similar constructions occur in [7] and [8]. Like their earlier relatives, the concordance invariants $f_{\sigma}$ defined in this paper provide lower bounds for the slice genus of a knot.

An intriguing feature of this construction in the instanton case is that, for suitably chosen $\sigma$, the concordance invariant may provide independent control of the genus and number of double points for normally immersed surfaces in the ball. (By normally immersed we shall mean that the the only self-intersection points of the immersed surface are transverse double points.) More specifically, for each $r \in[0,1]$, we can define a homomorphism

$$
f_{r}: \text { Conc } \rightarrow \mathbb{R}
$$

with the following property. Suppose $K$ is a knot in $S^{3}$ bounding a normally immersed, connected, oriented, surface $S \subset B^{4}$. Let $\gamma(S)$ be its genus, and $\varepsilon(S)$ the number of positive double points. Then the concordance invariant $f_{r}$ satisfies the inequality

$$
\gamma(S)+r \varepsilon(S) \geq f_{r}(K) .
$$

The authors' invariant $(1 / 2) s^{\sharp}(K)$ from [8] satisfies an inequality of this sort with $r=1$, as does the Ozsvath-Szabo $\tau$-invariant [10].

A priori, the new invariants with $r<1$ potentially constrain the ability to "trade handles for double-points" in immersed surfaces. Since any knot bounds a normally immersed disk, it is clear from the shape of (2) that $f_{r}(K) \rightarrow 0$ as $r \rightarrow 0$. So for small $r$ the inequality contains essentially no information about the genus of $S$. By considering a limiting case, we shall arrive also at a concordance homomorphism

$$
f_{*}: \text { Conc } \rightarrow \mathbb{R}
$$

with the property that, for a normally immersed disk $S$ in $B^{4}$ with boundary $K$, we have

$$
\varepsilon(S) \geq f_{*}(K) .
$$

The minimal number of crossings in a normally immersed disk - without concern for the signs of the crossings - is sometimes called the 4-dimensional clasp number or 4-dimensional crossing number of the knot $K$, and is often written $c_{*}(K)$. It follows that $\left|f_{*}(K)\right|$ is a lower bound for the 4-dimensional clasp number of a knot $K$ :

$$
c_{*}(K) \geq\left|f_{*}(K)\right|
$$


On the other hand, $\left|f_{*}(K)\right|$ may not be a lower bound for the slice genus of $K$. Remarks. As mentioned above, the association of an ideal $z^{\natural}(K)$ to a knot $K$ is formally similar also to the construction used by Alishahi and Eftekhary in [3], which is based on a variant of Heegaard-Floer homology for knots. Our invariants $f_{r}$, obtained from $z^{\natural}(K)$ by base-change to a suitable valuation ring, are similarly related in a formal way to the invariants $\Upsilon(t)$ defined by Ozsvath, Stipsicz and Szabo in [13], which one can derive from the Alishahi-Eftekhary invariants by a similar base change.

Although the authors hope to return to this in the future, the present paper contains no complete calculations of $z^{\natural}(K)$ or $f_{r}(K)$ for any non-trivial knots except the trefoil. For the simplest knots, such as 2-stranded torus knots, twist knots, and some small pretzel knots including the knot $7_{4}$ in the Rolfsen table, calculations of these invariants can be made based on just the formal properties that we establish. The results of some of these calculations are summarized at the end of this paper, but not included in detail, though we do include an alternative calculation for the trefoil to illustrate aspects of the gauge theory. The constructions in [3] also associate to a knot $K$ a module $\mathbb{A}(K)$ isomorphic to a monomial ideal in a ring of polynomials in two variables over a field of characteristic 2 . In the simplest cases that the authors have calculated, $z^{\natural}(K)$ agrees with $\mathbb{A}(K)$ after a base-change, but this appears to be a consequence of the fact that the two invariants share similar formal properties. For the torus knot $T(3,4)$, the authors believe that the two invariants are different. (See section 9.) One part of the difficulty in calculation arises from the fact we are working in characteristic 2 , where there is less prior work on instanton homology, though see $[17,16]$.

\section{Non-orientable surfaces}

There is a further formal similarity between the families of concordance homomorphisms $f_{\sigma}$ defined here and the invariants $\Upsilon(t),(0<t<2)$, defined by Ozsváth, Stipsicz and Szabó in [13]. Like $f_{\sigma}$, each $\Upsilon(t)$ is a homomorphism from the concordance group to $\mathbb{R}$. But in addition, it is shown in [14] that for the special case $t=1$, the invariant $\Upsilon(t)$ constrains the topology of non-orientable embedded surfaces $S \subset B^{4}$ with boundary $K$, by an inequality

$$
b_{1}(S)-\frac{1}{2} v(S) \geq-2 \Upsilon_{K}(1),
$$

where $v$ is the degree of the normal bundle of $S$ relative to the zero framing of the boundary. We shall see that certain specializations of the construction of $f_{\sigma}$ lead to concordance homomorphisms with the same property. 


\section{Crossing changes}

We shall also describe the behavior of $I^{\sharp}(K ; \Gamma)$ under crossing-changes of $K$. The rank of $I^{\sharp}(K ; \Gamma)$ over $\mathscr{R}$ is unchanged by crossing-changes, and only the torsion is affected. This is the same behavior as is described for $I^{\sharp}\left(K ; \Gamma_{o}\right)$ in the authors' earlier paper [8], which in turn rested on [7] and [6].

Corresponding results for Heegaard-Floer homology are proved in [12] and [3], and similar results for Bar-Natan homology and Lee homology were proved by Alishahi and Alishahi-Dowlin in [1,2]. As in [1, 2, 3], one can exploit the crossing-change behavior to show that the torsion part of $I^{\sharp}(K ; \Gamma)$ gives rise to a bound on the number of crossing changes needed to unknot $K$.

\section{Review of instanton homology with local coefficients}

\section{The basic construction}

We briefly recall some of the features of the instanton homology groups from [9]. Let $K$ be a link in a closed oriented 3-manifold $Y$, let $y_{0} \in Y$ be a framed basepoint and $B\left(y_{0}\right)$ a standard ball, disjoint from $K$. Let $\theta$ be a standard thetagraph embedded in $B\left(y_{0}\right)$, and let

$$
K^{\sharp}=K \cup \theta
$$

be the union, regarded as a web embedded in $Y$. We can equip $Y$ with the structure of an orbifold $Y$ whose singular set is $K^{\sharp}$ and whose local stabilizers are $\mathbb{Z} / 2$ along all edges of the web. There is then an associated space $\mathscr{B}^{\sharp}(Y, K)$ which parametrizes isomorphism classes of orbifold $S O(3)$ connections on $\breve{Y}$ equipped with a lift to $S U(2)$ on the complement of the singular set. The instanton homology $I^{\sharp}(Y, K)$ with coefficients $\mathbb{F}_{2}$ is constructed as the Morse homology of a perturbed Chern-Simons functional on $\mathscr{B}^{\sharp}(Y, K)$.

To define a system of local coefficients, one starts by constructing four maps

$$
h=\left(h_{0}, h_{1}, h_{2}, h_{3}\right): \mathscr{B}^{\sharp}(Y, K) \rightarrow(\mathbb{R} / \mathbb{Z})^{4} .
$$

The component $h_{0}$ is defined using the holonomy of a connection along all the components of the link $K$, while $h_{1}, h_{2}, h_{3}$ are defined using the holonomy along the edges of $\theta$. See [9]. The local system $\Gamma$ over $\mathscr{B}^{\sharp}(Y, K)$ is defined as the pullback via $h$ of a tautological local system over $(\mathbb{R} / \mathbb{Z})^{4}$ whose fiber is a free rank-1 module over the group ring $\mathscr{R}=\mathbb{F}_{2}\left[\mathbb{Z}^{4}\right]$. Formal variables $T_{i}$ are introduced so as to write $\mathscr{R}$ as the ring of finite Laurent series (1). 
Definition 2.1 ([9, section 2.2]). The instanton homology group of $(Y, K)$, denoted $I^{\sharp}(Y, K ; \Gamma)$, is the Floer homology group constructed from the perturbed Chern-Simons functional on $\mathscr{B}^{\sharp}(Y, K)$ with coefficients in the local system $\Gamma$. For any ring homomorphism of commutative rings, $\sigma: \mathscr{R} \rightarrow \mathcal{S}$, we write $\Gamma_{\sigma}$ for the local system $\Gamma \otimes_{\sigma} \mathcal{S}$, and $I^{\sharp}\left(Y, K ; \Gamma_{\sigma}\right)$ for the instanton homology.

Despite the appearance of the definition of $h_{0}$, a careful examination of the local system shows that the orientation of the link $K$ plays no role.

\section{A variant with non-zero Stiefel-Whitney class}

We also recall from [9] that given closed 1-manifold $\omega \subset Y$ disjoint from $K^{\sharp}$, there is a variant of $I^{\sharp}\left(Y, K ; \Gamma_{\sigma}\right)$ constructed from $S O(3)$ connections whose StiefelWhitney class is dual to $\omega$. More precisely, the space $\mathscr{B}^{\sharp}(Y, K)_{\omega}$ is defined as a space of orbifold $S O(3)$ connections on $\check{Y}$ together with a lift to $S U(2)$ on the complement of $K^{\sharp} \cup \omega$ and such that the obstruction to extending the lift across $\omega$ is -1 . The local system $\Gamma_{\sigma}$ can be defined on $\mathscr{B}^{\sharp}(Y, K)_{\omega}$ for any $\sigma$, and we have instanton homology groups

$$
I^{\sharp}\left(Y, K ; \Gamma_{\sigma}\right)_{\omega} .
$$

Rather than being a closed 1-manifold in the complement of the web, the locus $\omega$ can also be allowed to have components which are arcs with end-points on the link $K$. When $\omega$ has this form, the holonomy map $h_{0}$ can no longer be constructed using holonomy along $K$, and the local system $\Gamma$ is no longer defined. However, if $\sigma: \mathscr{R} \rightarrow \mathcal{S}$ is a base change with $\sigma\left(T_{0}\right)=1$, then $h_{0}$ plays no role in the definition of the local system $\Gamma_{\sigma}$. For such $\omega$, we may therefore define $I^{\sharp}\left(Y, K ; \Gamma_{\sigma}\right)_{\omega}$ whenever $\sigma\left(T_{0}\right)=1$.

\section{Functoriality for embedded cobordisms}

Having briefly reviewed the main features of the instanton homology groups $I^{\sharp}(Y, K ; \Gamma)$, we now turn to their functorial properties. Let $(X, S)$ be a cobordism from a pair $\left(Y_{0}, K_{0}\right)$ to a pair $\left(Y_{1}, K_{1}\right)$.We require as usual that $X$ is oriented so that

$$
\partial X=-Y_{0}+Y_{1}
$$

and in this section we will also require that $S$ is an oriented cobordism betweeen oriented links:

$$
\partial S=-K_{0}+K_{1}
$$


(This condition of orientability will be dropped later.) Because we wish to consider the instanton homology $I^{\sharp}$, we require standard embedded balls $B\left(y_{0}\right)$ and $B\left(y_{1}\right)$ at framed base-points $y_{0}$ and $y_{1}$, and an embedded $[0,1] \times B^{3}$ joining these in $X$ (see [9, Section 2.4]). We always require $S$ to be disjoint from these. However, we will usually not indicate them in our notation. The functoriality of instanton homology means that $S$ gives rise to a map of $\mathscr{R}$-modules,

$$
I^{\sharp}(X, S ; \Gamma): I^{\sharp}\left(Y_{0}, K_{0} ; \Gamma\right) \rightarrow I^{\sharp}\left(Y_{1}, K_{1} ; \Gamma\right) .
$$

This basic construction can be extended in various ways. First, without essential change, we can pass to a local system of $\delta$-modules $\Gamma_{\sigma}$ in place of $\Gamma$, by a base change $\sigma: \mathscr{R} \rightarrow \mathcal{S}$. Second, we can consider functoriality for the homology groups modified by a codimension- 2 representative $\omega$, as described in the previous paragraphs. Given a closed 1-manifolds $\omega_{i} \subset Y_{i}$ disjoint for $K_{i}^{\sharp}$ for $i=0,1$, and corresponding homology groups $I^{\sharp}\left(Y_{i}, K_{i} ; \Gamma_{\sigma}\right)_{\omega_{i}}$. Given a cobordism $(X, S)$ as before and also a 2-dimensional submanifold $\omega$ - a cobordism from $\omega_{0}$ to $\omega_{1}$, disjoint from $S$ - then one obtains a map

$$
I^{\sharp}(X, S ; \Gamma)_{\omega}: I^{\sharp}\left(Y_{0}, K_{0} ; \Gamma\right)_{\omega_{0}} \rightarrow I^{\sharp}\left(Y_{1}, K_{1} ; \Gamma\right)_{\omega_{1}} .
$$

As discussed in [9], one may allow $\omega$ to have transverse intersections with the interior of $S$ in $X$. Furthermore, in the special case that $\sigma\left(T_{0}\right)=1$, one may allow $\omega$ to be a manifold with corners whose boundary pieces are $\omega_{0}$ and $\omega_{1}$ together with arcs and circles on $S$.

Since $X$ will usually be fixed, and coefficients $\Gamma$ or $\Gamma_{\sigma}$ are understood, we may abbreviate the notation and just write, for example,

$$
I^{\sharp}(S)=I^{\sharp}\left(X, S ; \Gamma_{\sigma}\right) \text {. }
$$

\section{Immersed cobordisms}

We may allow the surface $S$ to be normally immersed (immersed with normal crossings) in $X$. To extend the definition of $I^{\sharp}\left(X, S ; \Gamma_{\sigma}\right)_{\omega}$ to this case, we first transform $S$ to an embedded surface by a blow-up construction: at each normal crossing, we replace $S$ by its proper transform $\tilde{S}$ in $\tilde{X}=X \# \overline{\mathbb{C P}}^{2}$. Following the convention about the immersed case that is captured by the formula To define this map in the case that $S$ is normally immersed rather than embedded, we first transform $S$ to an embedded surface by a blow-up construction. In the case of just a single double point, one then defines $I^{\sharp}(S)$ in terms of $I^{\sharp}(\tilde{S})$ by the rule:

$$
I^{\sharp}\left(X, S ; \Gamma_{\sigma}\right)_{\omega}=I^{\sharp}\left(\tilde{X}, \tilde{S} ; \Gamma_{\sigma}\right)_{\omega}+I^{\sharp}\left(\tilde{X}, \tilde{S} ; \Gamma_{\sigma}\right)_{\omega+\epsilon},
$$


where $\epsilon$ is the standard 2-sphere representing the generator of $H^{2}\left(\mathbb{C P}^{2}\right)$. For more than one double-point, one applies this construction repeatedly. See [9, Section 4.3] for details.

The reason for including both terms on the right hand side of (4) is explained in [9], and is appropriate when we wish to allow $\omega$ to have boundary along $S$. If $\omega$ and $\omega^{\prime}$ are two surfaces with boundary on $S$, and if they are isotopic by an isotopy in which $\partial \omega$ sweeps over a double-point of $S$ along one branch of the immersed surface, then the classes $\left[\omega^{\prime}\right]$ is homologous to $[\omega+\epsilon]$. Both terms are therefore needed if we wish to have a definition that is invariant under this isotopy. That said, if we allow only the more restricted representatives $\omega$ whose boundaries do not include arcs or circles on $S$, then this symmetry between the two terms is no longer needed. Following [9], we can therefore change the definition of the functor when applied to normally immersed surfaces. For any $\xi \in \mathcal{S}$ we may define a modified functor $I_{\xi}^{\sharp}$ by altering (4) to:

$$
I_{\xi}^{\sharp}\left(X, S ; \Gamma_{\sigma}\right)_{\omega}=I_{\xi}^{\sharp}\left(\tilde{X}, \tilde{S} ; \Gamma_{\sigma}\right)_{\omega}+\xi I_{\xi}^{\sharp}\left(\tilde{X}, \tilde{S} ; \Gamma_{\sigma}\right)_{\omega+\epsilon} \text {. }
$$

When applied to embedded surfaces, the functors $I_{\xi}^{\sharp}$ and $I^{\sharp}$ are equal.

\section{Surfaces with dots}

The functoriality can be extended by allowing the morphisms to be surfaces $S$ with additional decoration by dots: a dot is an interior point $q$ of $S$ together with an orientation of $T_{q} S$. Thus given $S$ and a dot $q$, there is an operator

$$
I^{\sharp}(S, q): I^{\sharp}\left(Y_{0}, K_{0} ; \Gamma\right) \rightarrow I^{\sharp}\left(Y_{1}, K_{1} ; \Gamma\right) .
$$

To define this extension, following section 3.4 of [9], it is sufficient to treat the case that $S$ is a trivial cobordism (a cylinder), in which case we are seeking to define an operator

$$
\Lambda_{q}: I^{\sharp}(Y, K ; \Gamma) \rightarrow I^{\sharp}(Y, K ; \Gamma) .
$$

This operator, which has even degree for the mod 2 grading, is defined in [9], where it is shown to satisfy a relation

$$
\Lambda_{q}^{2}+P \Lambda_{q}+Q=0
$$

where $P$ and $Q$ are elements of $\mathscr{R}$ given by

$$
P=T_{1} T_{2} T_{3}+T_{1} T_{2}^{-1} T_{3}^{-1}+T_{2} T_{3}^{-1} T_{1}^{-1}+T_{3} T_{1}^{-1} T_{2}^{-1}
$$


and

$$
Q=\sum_{j=0}^{3}\left(T_{j}^{2}+T_{j}^{-2}\right)
$$

\section{The ideal of a knot}

\section{Modifying surfaces in the orientable case}

There are standard ways in which an embedded or immersed surface $S$ can be modified by local operations to produce a new surface, and there are formulae proved in [9] for how the map $I^{\sharp}(S)$ may be changed such modifications. For the following definition, we refer to $[9,7]$ for a description of the twist moves and finger move. The "internal 1-handle addition" is illustrated in Figure 1. To describe it in words, points $p$ and $q$ lie on two disks in $S$, both of which are standardly embedded in a ball $B^{4} \subset X$. The new surface $S^{*}$ is obtained by replacing the two oriented disks with a standard oriented annulus. A special case of this operation is an internal connect sum with a standard torus.

Definition 3.1. Let $(X, S)$ and $\left(X, S^{*}\right)$ be cobordisms between the same pairs (so only the surface $S$ has changed). We continue to suppose that $S$ and $S^{*}$ are oriented. We will say that $S^{*}$ is c-equivalent to $S$ if $S^{*}$ can be obtained from $S$ by a sequence of moves, each of which is one of the following or its inverse:

- an ambient isotopy relative to the boundary;

- introducing a double point by a twist move, either positive or negative;

- introducing two new double points by a finger move;

- an oriented internal 1-handle addition connecting two points in the same connected component of the surface.

Remark. If $X$ is simply connected and $S \subset X$ is connected, then $c$-equivalence is the same as the equivalence relation generated by homotopy relative to the boundary together with "stabilization" by internal connected sum with $T^{2}$. For example, given two classical knots $K_{0}$ and $K_{2}$, any two connected, oriented cobordisms $S$ and $S^{*}$ joining them in $[0,1] \times S^{3}$ are $c$-equivalent.

Formulae for how $I^{\sharp}(S)$ changes when $S$ is changed by a twist move or a finger move were given in [9, Proposition 4.4]. The lemma below summarizes these, and 

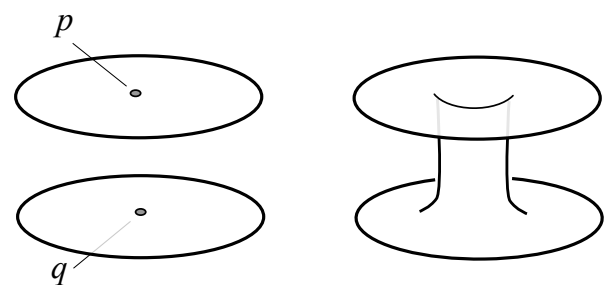

Figure 1: The internal addition of a handle. The surfaces are embedded in a standard 4-ball.

also provides a formula for internal 1-handle additions (a "neck-cutting relation" which generalizes the formula for the internal connected sum with a torus [9, Lemma 4.3]).

Lemma 3.2. (a) If $S^{\prime}$ is obtained from the oriented immersed cobordism $S$ by either a finger move or a positive twist move, then

$$
I^{\sharp}\left(S^{\prime}\right)=\sigma(L) I^{\sharp}(S)
$$

where

$$
L=P+T_{0}^{2}+T_{0}^{-2} \in \mathscr{R} .
$$

(b) If $S^{\prime}$ is obtained from $S$ by either a negative twist move, the $I^{\sharp}\left(S^{\prime}\right)=I^{\sharp}(S)$.

(c) If $S^{\prime}$ is obtained from the oriented cobordism $S$ by an internal 1-handle addition connecting two points $p$ and $q$ of $S$,

$$
I^{\sharp}\left(S^{\prime}\right)=I^{\sharp}(S, p)+I^{\sharp}(S, q)+P I^{\sharp}(S),
$$

where the notation $(S, p)$ means the surface $S$ decorated with a dot at $p$ using the orientation of $S$. In particular, if $p$ and $q$ are on the same component of $S$, then

$$
I^{\sharp}\left(S^{\prime}\right)=P I^{\sharp}(S) .
$$

Proof. It remains to prove the result for the internal 1-handle addition. Using excision, we can reduce the general case to the case that $S$ is the trivial cobordism from the 2-component unlink to itself. In this case, $S^{\prime}$ can be described as a composite of the "pants" and "copants" cobordisms, and $I^{\sharp}\left(S^{\prime}\right)$ can therefore be calculated using the results from section 5.4 of [9]. 
Two topological quantities associated to an oriented surface $S$ will be our focus here. The first is the number of positive double points, which we write as $\varepsilon(S)$. The second is the genus of $S$. In the context of cobordisms, it is convenient to make an adjustment to the genus:

Definition 3.3. For a cobordism $S$ from a link $K_{0}$ to a link $K_{1}$, we define the adjusted genus to be the quantity

$$
\gamma(S)=\left(-\chi(S)+c_{+}(S)-c_{-}(S)\right) / 2,
$$

where $c_{+}$and $c_{-}$are the number of components of the outgoing and incoming ends of of the cobordism (the number of components of $K_{1}$ and $K_{0}$ respectively).

Remark. If $K_{1}$ is a knot, then $\gamma(S)$ coincides with the usual genus of the surface, which is why there is no risk of confusion in using the same notation. The advantage of the adjusted genus is that it is additive for composite cobordisms.

With these definitions out of the way, we can state the main result from which the remainder of our conclusions will be derived.

Proposition 3.4. Suppose $S$ and $S^{*}$ are c-equivalent. Let $\gamma(S)$ and $\varepsilon(S)$ denote the adjusted genus and the number of positive double points. Then there exist $n$ and $m$ large enough such that

$$
P^{\gamma\left(S^{*}\right)+n} L^{\varepsilon\left(S^{*}\right)+m} I^{\sharp}(S)=P^{\gamma(S)+n} L^{\varepsilon(S)+m} I^{\sharp}\left(S^{*}\right) .
$$

Remark. If $I^{\sharp}\left(K_{1} ; \Gamma\right)$ is torsion-free and $\gamma(S), \gamma\left(S^{*}\right) \geq 0$, then there is no need for $n$ and $m$ in this proposition: the stated equality holds only if it already holds with $m=n=0$.

Proof of the Proposition. The essential calculations here are to show that the stated equality holds if $S^{*}$ is obtained from $S$ by just one of the moves in Definition 3.1 or their inverses. For such a single move, the result follow in each case from Lemma 3.2 (bearing in mind that Definition 3.1 requires that $p$ and $q$ are in the same component of $S$, in the case for an internal 1-handle addition). For more than one move, the result follows by induction.

\section{Definition of the ideal}

Let Frac $(\mathscr{R})$ denote the field of fractions of $\mathscr{R}$. If $M$ and $N$ are submodules of an $\mathscr{R}$-module $W$, we write $[N: M]$ for the generalized module quotient,

$$
[N: M]=\{a / b \in \operatorname{Frac}(\mathscr{R}) \mid a M \subset b N\} .
$$


This is an $\mathscr{R}$-submodule of $\operatorname{Frac}(\mathscr{R})$. In this definition, if the modules might have torsion, we should allow $a / b$ to be a fraction that is not expressed in lowest terms.

Definition 3.5. Given a cobordism $(X, S)$ as above from $\left(Y_{0}, K_{0}\right)$ to $\left(Y_{1}, K_{1}\right)$, we define the $\mathscr{R}$-submodule

$$
\zeta(X, S) \subset \operatorname{Frac}(\mathscr{R})
$$

to be $\zeta(X, S)=[N: M]$, where $N=I^{\sharp}\left(Y_{1}, K_{1} ; \Gamma\right)$ and $M \subset N$ is the image of the $\mathscr{R}$-module homomorphism $I^{\sharp}(S ; \Gamma)$ :

$$
M=\operatorname{im} I^{\sharp}(S ; \Gamma) .
$$

Further, if $\gamma$ and $\varepsilon$ are the adjusted genus and number of positive double points in $S$, we define

$$
\begin{aligned}
z^{\sharp}(X, S) & =P^{\gamma} L^{\varepsilon} \zeta(X, S) \\
& \subset \operatorname{Frac}(\mathscr{R}) .
\end{aligned}
$$

Note that $1 \in \zeta(X, S)$, so $z^{\sharp}(X, S)$ is always a non-zero submodule of $\operatorname{Frac}(\mathscr{R})$.

Lemma 3.6. If $S^{*} \subset X$ is c-equivalent to $S$, then the $\mathscr{R}$-submodules $z^{\sharp}(X, S)$ and $z^{\sharp}\left(X, S^{*}\right)$ in $\operatorname{Frac}(\mathscr{R})$ are equal.

Proof. This follows from the definition and Proposition 3.4.

Corollary 3.7. If $S$ is c-equivalent to a surface $S^{*}$ with adjusted genus $\gamma^{*}$ and $\varepsilon^{*}$ positive double points (and any number of negative double points), then

$$
P^{\gamma^{*}} L^{\varepsilon^{*}} \in z^{\sharp}(X, S) .
$$

Proof. Given Proposition 3.4, this again follows by checking the definitions. To do this, let $M$ be the image of $I^{\sharp}(S)$ and let $M_{*}$ be the image of $I^{\sharp}\left(S^{*}\right)$. Since $M_{*}$ is certainly contained in $I^{\sharp}\left(Y_{1}, K_{1} ; \Gamma\right)$, we evidently have

$$
r P^{\gamma} L^{\varepsilon} M_{*} \subset r P^{\gamma} L^{\varepsilon} I^{\sharp}\left(Y_{1}, K_{1} ; \Gamma\right) .
$$

From Proposition 3.4, with $r=P^{n} L^{m}$, we have

$$
r P^{\gamma} L^{\varepsilon} M_{*}=r P^{\gamma^{*}} L^{\varepsilon_{*}} M,
$$

so the previous inclusion also says

$$
r P^{\gamma^{*}} L^{\varepsilon_{*}} M \subset r P^{\gamma} L^{\varepsilon} I^{\sharp}\left(Y_{1}, K_{1} ; \Gamma\right) .
$$


From the definition of $\zeta(X, S)$, this last inclusion means

$$
P^{\gamma^{*}-\gamma} L^{\varepsilon_{*}-\varepsilon} \in \zeta(X, S)
$$

which is equivalent to

$$
P^{\gamma^{*}} L^{\mathcal{E}_{*}} \in z^{\sharp}(X, S) .
$$

Lemma 3.8. If $X$ is a product, $[0,1] \times Y$, and $S$ is c-equivalent to a product $[0,1] \times K$, then $z^{\sharp}(X, S)=\mathscr{R}$.

Proof. Because of Lemma 3.6, we may as well assume $S$ is product. In this case, the map is the identity, so $\zeta(X, S)=\mathscr{R}$ by construction. On the other hand, $\gamma$ and $\varepsilon$ are both zero for a product cobordism, so $z^{\sharp}(X, S)=\zeta(X, S)$.

Lemma 3.9. Suppose $\left(X_{i}, S_{i}\right)$ is a cobordism from $\left(Y_{i-1}, K_{i-1}\right)$ to $\left(Y_{i}, K_{i}\right)$, for $i=1,2$. Let $(X, S)$ be the composite cobordism. Then

$$
z^{\sharp}\left(X_{1}, S_{1}\right) z^{\sharp}\left(X_{2}, S_{2}\right) \subset z^{\sharp}(X, S) .
$$

Proof. The terms $\gamma(S)$ and $\varepsilon(S)$ are both additive, so the assertion is equivalent to

$$
\zeta\left(X_{1}, S_{1}\right) \zeta\left(X_{2}, S_{2}\right) \subset \zeta(X, S) .
$$

For brevity, write $N_{i}=I^{\sharp}\left(Y_{i}, K_{i} ; \Gamma\right)$ for $i=0,1,2$. If $a_{1} / b_{1} \in \zeta\left(X_{1}, S_{1}\right)$ and $a_{2} / b_{2} \in$ $\zeta\left(X_{2}, S_{2}\right)$, then

$$
\begin{aligned}
\left(a_{1} a_{2}\right) \operatorname{im}\left(I^{\sharp}(S)\right) & =a_{2} I^{\sharp}\left(S_{2}\right)\left(a_{1} \operatorname{im}\left(I^{\sharp}\left(S_{1}\right)\right)\right. \\
& \subset a_{2} I^{\sharp}\left(S_{2}\right)\left(b_{1} N_{1}\right) \\
& =b_{1} a_{2} \operatorname{im} I^{\sharp}\left(S_{2}\right) \\
& \subset b_{1} b_{2} N_{2} .
\end{aligned}
$$

So $\left(a_{1} a_{2}\right) /\left(b_{1} b_{2}\right) \in \zeta(X, S)$ as required.

Corollary 3.10. Let $\left(X_{1}, S_{1}\right)$ be a cobordism from $\left(Y_{0}, K_{0}\right)$ to $\left(Y_{1}, K_{1}\right)$. Suppose there exists $\left(X_{2}, S_{2}\right)$ such that the composite $(X, S)$ is c-equivalent to a product. That is, $X=X_{1} \cup X_{2}$ is diffeomorphic to a cylinder $[0,1] \times Y_{0}$ by a diffeomorphism $h$, and $h(S)$ is c-equivalent to $[0,1] \times K_{0}$. Then $z^{\sharp}\left(X_{1}, S_{1}\right)$ is a fractional ideal, meaning there exists $A \in \mathscr{R}$ such that

$$
A z^{\sharp}\left(X_{1}, S_{1}\right) \subset \mathscr{R} \text {. }
$$


Proof. From the previous three lemmas, we have

$$
z^{\sharp}\left(X_{1}, S_{1}\right) z^{\sharp}\left(X_{2}, S_{2}\right) \subset \mathscr{R} .
$$

The submodule $z^{\sharp}\left(X_{2}, S_{2}\right)$ is non-zero (as always) so contains some non-zero $A / B \in \operatorname{Frac}(\mathscr{R})$. Then the above inclusion gives

$$
A z^{\sharp}\left(X_{1}, S_{1}\right) \subset B \mathscr{R} \text {. }
$$

\section{Classical knots}

Let us focus now on the special case of classical knots in $S^{3}$, and take $X=[0,1] \times$ $S^{3}$. Consider connected cobordisms $S$ from the unknot $U_{1}$ to a general knot $K$. Any two such cobordisms are c-equivalent. Furthermore, Corollary 3.10 always applies in this situation. So we can make the following definition.

Definition 3.11. For a classical knot $K$, we define $z^{\sharp}(K) \subset \operatorname{Frac}(\mathscr{R})$ to be the fractional ideal $z^{\sharp}(X, S)$, where $X=[0,1] \times S^{3}$, and $S$ is any connected, oriented cobordism from $U_{1}$ to $K$. This fractional ideal is independent of the choice of $S$.

Remarks. In the situation described in this definition, we can construct a cobor$\operatorname{dism} S^{\prime}=D^{2} \cup S$ from the empty link $U_{0}$ to $K$, and we can equivalently define $z^{\sharp}(K)$ to be $z^{\sharp}\left(X, S^{\prime}\right)$. To see that these are equal, note first that $\operatorname{im} I^{\sharp}\left(S^{\prime}\right) \subset \operatorname{im} I^{\sharp}(S)$, so an inclusion

$$
z^{\sharp}(X, S) \subset z^{\sharp}\left(X, S^{\prime}\right)
$$

follows from the definition. To obtain equality, note that there is the point operator $\Lambda=\Lambda_{p}$ acting on both $I^{\sharp}\left(U_{1} ; \Gamma\right)$ and $I^{\sharp}(K ; \Gamma)$, so $I^{\sharp}(S)$ is a homomorphism of modules over the larger ring $\mathscr{F}=\mathscr{R}[\Lambda] /\left(\Lambda^{2}+P \Lambda+Q\right)$. The Floer homology $I^{\sharp}\left(U_{1} ; \Gamma\right)$ is a free module of rank 1 over $\mathscr{F}$, generated by $\mathbf{x}_{+}$, and the latter element is in the image of the map

$$
I^{\sharp}\left(D^{2}\right): I^{\sharp}\left(U_{0} ; \Gamma\right) \rightarrow I^{\sharp}\left(U_{1} ; \Gamma\right) .
$$

So $\zeta(X, S)$ and $\zeta\left(X, S^{\prime}\right)$ can both be described as the set of $a / b$ such that

$$
a I^{\sharp}(S)\left(\mathbf{x}_{+}\right) \in b I^{\sharp}(K ; \Gamma) .
$$

From the fractional ideal $z^{\sharp}(K)$, we can read off a constraint on the possible genus and number of positive double points, for surfaces $S$ in $B^{4}$ which bound $K$. 
Proposition 3.12. If the classical knot $K \subset S^{3}$ bounds a surface $S$ in $B^{4}$ with genus $\gamma$ and $\varepsilon$ positive double points (and any number of negative double points), then

$$
P^{\gamma} L^{\varepsilon} \in z^{\sharp}(K) .
$$

Proof. This follows from the definition and Corollary 3.7.

As a generalization of the above proposition, we have the following.

Proposition 3.13. Let $S$ be a normally immersed cobordism from a knot $K_{0}$ to a $k n o t K_{1}$. Let $\gamma$ be its genus and $\varepsilon$ the number of positive double points. Then

$$
P^{\gamma} L^{\varepsilon} z^{\sharp}\left(K_{0}\right) \subset z^{\sharp}\left(K_{1}\right) \text {. }
$$

Proof. Let $S_{0}$ be a cobordism from the unknot to $K_{0}$, and let $S_{1}$ be the composite of $S_{0}$ and $S$, from the unknot to $K_{1}$. From Lemma 3.9 we have

$$
z^{\sharp}\left(X, S_{0}\right) z^{\sharp}(X, S) \subset z^{\sharp}\left(X, S_{1}\right),
$$

where $X$ is $[0,1] \times S^{3}$ in each case. From Corollary 3.7, we have $P^{\gamma} L^{\varepsilon} \in z^{\sharp}(X, S)$. So the above inclusion implies

$$
P^{\gamma} L^{\varepsilon} z^{\sharp}\left(X, S_{0}\right) \subset z^{\sharp}\left(X, S_{1}\right),
$$

which is to say, $P^{\gamma} L^{\varepsilon} z^{\sharp}\left(K_{0}\right) \subset z^{\sharp}\left(X, K_{1}\right)$ as claimed.

Corollary 3.14. For a classical knot $K$, the fractional ideal $z^{\sharp}(K) \subset \operatorname{Frac}(\mathscr{R})$ is a concordance invariant of $K$.

Proof. We apply the previous proposition to a concordance from $K_{0}$ to $K_{1}$, and we see $z^{\sharp}\left(K_{0}\right) \subset z^{\sharp}\left(K_{1}\right)$. The reverse inclusion holds for the same reason.

We make some remarks about the concordance invariant $z^{\sharp}(K) \subset \operatorname{Frac}(\mathscr{R})$, which seems to be of interest. Previous constraints on embedded surfaces that have been obtained using gauge theory have most often treated genus and positive double-points even-handedly. Thus the results of [6] lead to a lower bound on $\gamma(S)+\varepsilon(S)$, for embedded surfaces $S$ in a fixed homology class in a simplyconnected 4-manifold. The closely-related knot invariant $s^{\sharp}(K)$ defined in [8] is a cousin of Rasmussen's $s$-invariant for knots, and has the property that

$$
\gamma(S)+\varepsilon(S) \geq s^{\sharp}(K) / 2,
$$


for any oriented immersed surface in $S \subset B^{4}$ with boundary the knot.

By contrast with the invariant $s^{\sharp}(K)$, the invariant $z^{\sharp}(K)$ appears to have the potential to provide a constraint on the pair $(\gamma(S), \varepsilon(S))$ which is not a constraint only on their sum. Our results say that the pair is constrained to lie in the set

$$
G(K)=\left\{(\gamma, \varepsilon) \in \mathbb{N} \times \mathbb{N} \mid P^{\gamma} L^{\varepsilon} \in z^{\sharp}(K)\right\} .
$$

That said, the authors lack any resources for calculating $z^{\sharp}(K)$, except in some simple examples, at least at the time of writing. By smoothing a double point, one can always decrease $\varepsilon$ by one (if it is positive) in exchange for increasing $\gamma$ by one. That is, $(\gamma, \varepsilon)$ arises as the genus and number of double points for an immersed surface, then so does $(\gamma+1, \varepsilon-1)$, if $\varepsilon>0$. It would be interesting to know whether the set $G(K)$ shares this property.

\section{Non-orientable surfaces}

\section{Adaptation of the ideal to the non-orientable case}

As mentioned in the previous paragraph, if $\sigma: \mathscr{R} \rightarrow \mathcal{S}$ is a base change, then we can repeat the constructions above, with $\Gamma_{\sigma}=\Gamma \otimes_{\sigma} \mathcal{S}$ replacing the local system $\Gamma$, and $\mathcal{S}$-modules replacing $\mathscr{R}$-modules in the discussion throughout. We require only that $\mathcal{S}$ is an integral domain and that $\sigma(P)$ and $\sigma(L)$ are both non-zero. So, to a cobordism $(X, S)$ as above, we can associate an $\mathcal{S}$-module,

$$
z_{\sigma}^{\sharp}(X, S) \subset \operatorname{Frac}(\mathcal{S}),
$$

in the field of fractions $\operatorname{Frac}(\mathcal{S})$ of $\mathcal{S}$. (The condition that $\sigma(P)$ and $\sigma(L)$ are nonzero is used, for example, in the proof of Lemma 3.6.) For a classical knot $K$, this provides a fractional ideal

$$
z_{\sigma}^{\sharp}(K)
$$

which is again a concordance invariant of the knot. Proposition 3.12 continues to hold, and tells us that if $K$ bounds an oriented surface $S$ with adjusted genus $\gamma$ and $\varepsilon$ positive double points, then

$$
\sigma(P)^{\gamma} \sigma(L)^{\varepsilon} \in z_{\sigma}^{\sharp}(K) .
$$

In this form, nothing is gained from the base change: the above constraint on $\gamma$ and $\varepsilon$ can only be weaker than the previous one. However, there is a special class of cases in which $z_{\sigma}^{\sharp}(K)$ contains information also about non-orientable 
surfaces. We suppose from now on in this section that

$$
\sigma\left(T_{0}\right)=1 \in \mathcal{S} .
$$

This means in particular that $\sigma(P)=\sigma(L)$, so the constraint (9) becomes

$$
\sigma(P)^{\gamma+\varepsilon} \in z_{\sigma}^{\sharp}(K) \text {. }
$$

Consider now a possibly non-orientable immersed surface $S$ in $B^{4}$, with boundary the classical knot $K \subset S^{3}$. We continue to define $\gamma(S)$ as before (Definition 3.3), and we call it still the adjusted genus. We do not have a notion of positive or negative double point any more, so we simply write

$$
\delta(S)=\text { number of double points . }
$$

In addition to $\gamma$ and $\delta$, there is one other numerical invariant to record, which is the degree of the immersed normal bundle of $S$, relative to the trivialization at $\partial S$ provided by the 0 -framing of $K$. We write this as

$$
v(S)=\operatorname{deg} N S \text {. }
$$

For an orientable surface, this can already be non-zero if $S$ has double points but is zero if $S$ is embedded. If $S$ is non-orientable, it may be non-zero even for an embedded surface. We combine these and define

$$
\eta(S)=\gamma(S)+\frac{1}{2} \delta(S)-\frac{1}{4} v(S)
$$

This quantity is an integer, as will emerge below. For an orientable surface, we have

$$
\eta(S)=\gamma(S)+\varepsilon(S)
$$

so the constraint (9) can be rewritten yet again as

$$
\sigma(P)^{\eta(S)} \in z_{\sigma}^{\sharp}(K) .
$$

We now have the following theorem.

Theorem 4.1. Let $\sigma: \Re \rightarrow \mathcal{R}$ be a base-change with $\sigma\left(T_{0}\right)=1$, let $K \subset S^{3}$ be a knot, and let $z_{\sigma}^{\sharp}(K) \subset \operatorname{Frac}(\mathcal{S})$ be the associated fractional ideal as above. Let $S$ be a possibly non-orientable, normally immersed connected surface in $B^{4}$, with boundary K. Then

$$
\sigma(P)^{\eta(S)} \in z_{\sigma}^{\sharp}(K),
$$

where $\eta(S)$ is as in (10).

Note that in the statement of this theorem, the definition of $z_{\sigma}^{\sharp}(K)$ has not changed, and is still obtained by using an orientable surface, as in Definition 3.11. 


\section{Proof of the Theorem for the non-orientable case}

The proof of the theorem follows the same basic plan as the proof of Proposition 3.12 which treats the orientable case. The proof of that proposition arose from considering the effect of altering an immersed surface $S$ by finger moves, twist moves, and the addition of handles. For the non-orientable case, we consider also the effect of a connect sum with $\mathbb{R P}^{2}$, as in [9, Lemma 4.2].

To carry this out, consider the immersed cobordism in $I \times S^{3}$,

$$
S^{*}: U_{1} \rightarrow K
$$

obtained by removing a standard pair $\left(B_{\epsilon}^{4}, D_{\epsilon}^{2}\right)$ from $\left(B^{4}, S\right)$. Let $\omega$ be an immersed surface in the interior of $I \times S^{3}$ whose boundary is a collection of simple closed curves $\partial \omega \subset \operatorname{int}\left(S^{*}\right)$, along which $\omega$ and $S^{*}$ meet cleanly. Let $\omega$ be chosen furthermore so that the curves $\partial \omega$ is a representative for the Poincare dual of $w_{1}\left(S^{*}\right)$ in $H_{1}\left(S^{*} ; \mathbb{Z} / 2\right)$ :

$$
\operatorname{PD}_{S^{*}}[\partial \omega]=w_{1}\left(S^{*}\right)
$$

The relative homology class of $\omega$ is uniquely characterized by this condition. Corresponding to the cobordism $S^{*}$ and the surface $\omega$, we have a homomorphism

$$
I^{\sharp}\left(S^{*} ; \Gamma_{\sigma}\right)_{\omega}: I^{\sharp}\left(U_{1} ; \Gamma_{\sigma}\right) \rightarrow I^{\sharp}\left(K ; \Gamma_{\sigma}\right) .
$$

The homomorphism is independent of the choice of $\omega$, subject to the constraint (12), because it depends only on the relative homology class. Note that the particular choice we made in (4) for how to define $\left.I^{\sharp}\left(S^{*} ; \Gamma_{\sigma}\right)_{\omega}\right)$ in the case of immersed rather than embedded surfaces is important at this point. As explained in the remarks there, the symmetry between the two terms on the right-hand side of (4) is necessary to ensure that $I^{\sharp}\left(S^{*} ; \Gamma_{\sigma}\right)_{\omega}$ is unchanged if $\omega$ is modified by an isotopy that moves $\partial \omega$ across one of the double-points of the surface.

Let $S_{0}^{*}$ be any other immersed cobordism with the same boundary. As immersed surfaces in $I \times S^{3}$, these two differ by a sequence of operations each of which is one of the following or its inverse:

- an ambient isotopy relative to the boundary;

- introducing a double point by a twist move, either positive or negative;

- introducing two new double points by a finger move; 
- an internal connected sum with an embedded $\mathbb{R P}^{2}$ of the sort $R_{+}$, as in [9, Lemma 4.2]. (Recall that $R_{+}$is an embedded $\mathbb{R} \mathbb{P}^{2}$ with self-intersection +2 .)

Remark. It is not necessary to include a connect sum with $R_{-}$in this list, because the same effect can be achieved by a sum with $R_{+}$followed by isotopies, finger moves, and twist moves. Similarly, it is not necessary to include a sum with $T^{2}$.

So let $S_{0}^{*}, S_{1}^{*}, \ldots, S_{k}^{*}=S^{*}$ be a sequence of surfaces related each to the next by one of these operations or its inverse. For each $S_{j}^{*}$, let $\omega_{j}$ be an immersed surface in the interior of $I \times S^{3}$ whose boundary $\partial \omega_{j} \subset \operatorname{int}\left(S_{j}^{*}\right)$ is dual to $w_{1}\left(S_{j}^{*}\right)$ For each $j$, consider the resulting homomorphism,

$$
I^{\sharp}\left(S_{j}^{*} ; \Gamma_{\sigma}\right)_{\omega_{j}}: I^{\sharp}\left(U_{1} ; \Gamma_{\sigma}\right) \rightarrow I^{\sharp}\left(K ; \Gamma_{\sigma}\right) .
$$

Consider one step in this sequence: suppose that $S_{1}^{*}$ is obtained from $S_{0}^{*}$ by one of the operations listed above. In the case of the twist move and finger move, we can suppose that $\omega_{0}$ is disjoint from the regions involved in the modification of $S_{0}$, and we can take $\omega_{1}=\omega_{0}$. The situation then is no different from the orientable case, and accordingly we have

$$
I^{\sharp}\left(S_{1}^{*} ; \Gamma_{\sigma}\right)_{\omega_{1}}=U I^{\sharp}\left(S_{0}^{*} ; \Gamma_{\sigma}\right)_{\omega_{0}},
$$

where

$$
U= \begin{cases}\sigma(P), & \text { for the finger and positive twist moves, } \\ 1, & \text { for the negative twist move. }\end{cases}
$$

In the case that $S_{1}^{*}=S_{0}^{*} \# R_{+}$, in order to satisfy the constraint (12), we can take $\omega_{1}$ to be $\omega_{0} \cup \pi$, where $\pi$ is a disk meeting $R_{+}$in a generator of $H_{1}\left(R_{+}\right)$. According to [9, Lemma 4.2], we then have

$$
I^{\sharp}\left(S_{1}^{*} ; \Gamma_{\sigma}\right)_{\omega_{1}}=I^{\sharp}\left(S_{0}^{*} ; \Gamma_{\sigma}\right)_{\omega_{0}} .
$$

At the same time, we can consider how the numerical invariant $\eta(S)$ is changed by these operations. For the finger move, $v(S)$ is unchanged, while $\delta(S)$ increases by 2 . For the positive (respectively, negative) twist moves, $v(S)$ decreases (respectively, increases) by 2 . So we have

$$
\eta\left(S_{1}\right)=\eta\left(S_{0}\right)+\tau,
$$

where

$$
\tau= \begin{cases}1, & \text { for the finger and positive twist moves } \\ 0, & \text { for the negative twist move }\end{cases}
$$


For the sum with $R_{+}$, the adjusted genus $\gamma(S)$ increases by $1 / 2$, and $v(S)$ increases by 2 , so

$$
\eta\left(S_{1}\right)=\eta\left(S_{0}\right)
$$

in this case. (Note in particular that the change in $\eta$ is always an integer, which allows us to verify that $\eta(S) \in \mathbb{Z}$, as $\eta\left(S_{0}\right)$ is manifestly an integer if $S_{0}$ is orientable.)

If we compare the formulae for the change in $\eta(S)$ with the formulae for the change in $I^{\sharp}\left(S ; \Gamma_{\sigma}\right)_{\omega}$, we see that

$$
\sigma(P)^{\eta\left(S_{0}\right)} I^{\sharp}\left(S_{1}^{*} ; \Gamma_{\sigma}\right)_{\omega_{1}}=\sigma(P)^{\eta\left(S_{1}\right)} I^{\sharp}\left(S_{0}^{*} ; \Gamma_{\sigma}\right)_{\omega_{0}} .
$$

If we apply this argument to the sequence of modifications from $S_{0}$ to $S$, we learn that for some $n \geq 0$,

$$
\sigma(P)^{\eta\left(S_{0}\right)+n} I^{\sharp}\left(S^{*} ; \Gamma_{\sigma}\right)_{\omega}=\sigma(P)^{\eta(S)+n} I^{\sharp}\left(S_{0}^{*} ; \Gamma_{\sigma}\right)_{\omega_{0}} .
$$

From here, the proof of the theorem proceeds exactly as in the orientable case, which is the case already established at (11).

\section{More general non-orientable cobordisms}

As in the orientable case, the above theorem for classical knots can be set up more generally for cobordisms of pairs $(Y, K)$. Although we will not spell this out in full, we can usefully describe the appropriate functorial setup. For this purpose, we need to keep track not just of the surface $S$ in a morphism, but also the surface $\omega$. In more detail, the correct category has objects $\left(Y_{0}, K_{0}\right)$ and $\left(Y_{1}, K_{1}\right)$, where $Y_{i}$ is a closed oriented 3-manifold, and $K_{i} \subset Y_{i}$ is an oriented link. Again, $B\left(y_{i}\right) \subset Y_{i}$ will be a standard ball disjoint from $K_{i}$, a neighborhood of a chosen basepoint. For a morphism from from $\left(Y_{0}, K_{0}\right)$ to $\left(Y_{1}, K_{1}\right)$ we require the following data:

(a) a cobordism of pairs $(X, S)$, with $X$ an oriented 4-manifold and $S$ a (possibly non-orientable) immersed surface with transverse double-points;

(b) a surface $\omega$ in the interior of $X$ whose boundary is a collection of simple closed curves $\partial \omega \subset \operatorname{int}(S)$, along which $\omega$ and $S$ meet cleanly;

(c) an orientation of $S \backslash \partial \omega$ which is compatible with the orientations $-K_{0}$ and $K_{1}$ at the boundary, and which changes sign across the curves $\partial \omega$;

(d) an embedded cylinder $[0,1] \times B^{3}$ (or framed arc) joining $B\left(y_{0}\right)$ to $B\left(y_{1}\right)$, disjoint from $S$ and $\omega$. 
The orientation conditions imply that $\partial \omega$ represents to the dual of $w_{1}(S)$. We will say that two cobordisms $(X, S, \omega)$ and $\left(X^{\prime}, S^{\prime}, \omega^{\prime}\right)$ are isomorphic if they are diffeomorphic relative to the boundary, respecting orientations. Set up in this way, morphisms compose correctly. From $I^{\sharp}$ we obtain a functor which assigns $I^{\sharp}\left(Y, K ; \Gamma_{\sigma}\right)$ to the object $(Y, K)$, and assigns $I^{\sharp}\left(X, S ; \Gamma_{\sigma}\right)_{\omega}$ to the morphism $(X, S, \omega)$ as expected.

Imitating the previous definitions used in the orientable case, we can now formulate the following generalization of $z^{\sharp}(X, S)$ (Definition 3.5). Given a morphism $(X, S, \omega)$ as just described, let $M$ be the image of $I^{\sharp}\left(X, S ; \Gamma_{\sigma}\right)_{\omega}$, and let

$$
\zeta_{\sigma}(X, S, \omega) \subset \operatorname{Frac}(\delta)
$$

be the $\mathcal{S}$-submodule

$$
\zeta_{\sigma}(X, S, \omega)=\left[I^{\sharp}\left(Y_{1}, K_{1} ; \Gamma_{\sigma}\right): M\right] .
$$

Then set

$$
\begin{aligned}
z_{\sigma}^{\sharp}(X, S, \omega) & =\sigma(P)^{\eta(S)} \zeta_{\sigma}(X, S, \omega) \\
& \subset \operatorname{Frac}(S),
\end{aligned}
$$

again as in Definition 3.5. The proof of Theorem 4.1 adapts readily to establish that the submodule $z_{\sigma}^{\sharp}(X, S, \omega)$ is unchanged if $S$ and $\omega$ are altered by certain standard operations. To spell this out, let us say that $(S, \omega)$ and $\left(S^{\prime}, \omega^{\prime}\right)$ are $\tilde{c}^{-}$ equivalent if one can be obtained from the other by a sequence of the following moves and their inverses:

- an ambient isotopy relative to the boundary;

- replacing $\omega$ with another homologous surface;

- altering $S$ by introducing a double point by a twist move, either positive or negative, in 4-ball disjoint from $\omega$;

- introducing two new double points by a finger move, in a ball disjoint from $\omega$;

- replacing $(S, \omega)$ by $\left(S \# R_{+}, \omega \cup \pi\right)$, where $R_{+} \subset S^{4}$ is a standard $\mathbb{R} \mathbb{P}^{2}$ as before, and $\pi$ is a disk in $S^{4}$ whose boundary is a generating circle in $R_{+}$.

With these definitions, the statement becomes: 
Corollary 4.2. If $\sigma\left(T_{0}\right)=1$ and $(S, \omega)$ is $\tilde{c}$-equivalent to $\left(S^{*}, \omega^{*}\right)$ then the corresponding modules $z_{\sigma}^{\sharp}(X, S, \omega)$ and $z_{\sigma}^{\sharp}\left(X, S^{*}, \omega^{*}\right)$ are equal.

As a consequence, we have a lower bound on $\eta\left(S^{*}\right)$ for any $\tilde{c}$-equivalent pair (cf. Corollary 3.7):

Corollary 4.3. If $\sigma\left(T_{0}\right)=1$ and $(S, \omega)$ is $\tilde{c}$-equivalent to $\left(S^{*}, \omega^{*}\right)$, then

$$
\sigma(P)^{\eta\left(S^{*}\right)} \in z_{\sigma}^{\sharp}(X, S, \omega) \text {. }
$$

\section{Reduced homology and concordance homomorphisms}

\section{Using reduced homology}

Recall from [9, section 2.5] that if $\sigma: \mathscr{R} \rightarrow \mathcal{S}$ is a base-change with $\sigma\left(T_{0}\right)=$ $\sigma\left(T_{1}\right)$, then there is a reduced variant $I^{\natural}\left(K ; \Gamma_{\sigma}\right)$ of the corresponding instanton homology. If we continue to suppose that $\delta$ is at least an integral domain and $\sigma(P)$ and $\sigma(L)$ are non-zero, then we can use $I^{\natural}$ in place of $I^{\sharp}$ to define a fractional ideal

$$
z_{\sigma}^{\natural}(K) \subset \operatorname{Frac}(\delta)
$$

as a variant of $z_{\sigma}^{\sharp}(K)$. For the case of a knot $K$, this is algebraically a little simpler than $z_{\sigma}^{\sharp}(K)$. In this case, the instanton homology $I^{\natural}\left(K ; \Gamma_{\sigma}\right)$ has rank 1 . If we write

$$
I^{\natural}\left(K ; \Gamma_{\sigma}\right)^{\prime}=I^{\natural}\left(K ; \Gamma_{\sigma}\right) /(\text { Torsion }),
$$

then, being a finitely-generated, rank-1 torsion-free module over $\delta$, this quotient is isomorphic to an ideal $\mathscr{f}_{K}$ of $\mathcal{S}$ (though not uniquely). Choose such an isomorphism of $\mathcal{S}$-modules,

$$
\phi: I^{\natural}\left(K ; \Gamma_{\sigma}\right)^{\prime} \rightarrow \mathscr{I}_{K}
$$

If $S$ is a cobordism of based knots, from a knot $K_{0}$ to $K_{1}$, then we have a homomorphism

$$
I^{\natural}\left(S ; \Gamma_{\sigma}\right)^{\prime}: I^{\natural}\left(K_{0} ; \Gamma_{\sigma}\right)^{\prime} \rightarrow I^{\natural}\left(K_{1} ; \Gamma_{\sigma}\right)^{\prime},
$$

and in the special case of a cobordism from the unknot $U_{1}$ to $K$,

$$
I^{\natural}\left(S ; \Gamma_{\sigma}\right)^{\prime}: S \rightarrow I^{\natural}\left(K_{1} ; \Gamma_{\sigma}\right)^{\prime} .
$$


Let $\iota$ be the image of 1 under $I^{\natural}\left(S ; \Gamma_{\sigma}\right)^{\prime}$. The reduced version of $\zeta_{\sigma}$ in this situation is

$$
\begin{aligned}
\zeta_{\sigma}^{\natural}(K) & =\left[I^{\natural}\left(S ; \Gamma_{\sigma}\right): S_{l}\right] \\
& =\phi(\iota)^{-1} \mathscr{F}_{K} .
\end{aligned}
$$

In particular, the fractional ideal $\zeta_{\sigma}^{\natural}(K)$ is isomorphic to $I^{\natural}\left(K ; \Gamma_{\sigma}\right)^{\prime}$ as a $\mathcal{S}$-module. The concordance invariant is the fractional ideal

$$
z_{\sigma}^{\natural}(K)=\sigma(P)^{\gamma} \sigma(L)^{\varepsilon} \zeta_{\sigma}^{\natural}(K)
$$

(Definition 3.5), which is therefore also isomorphic to $I^{\natural}\left(K ; \Gamma_{\sigma}\right)^{\prime}$ as a $\mathcal{S}$-module.

For the case of reduced homology of a knot, a somewhat more direct definition of the ideal $z_{\sigma}^{\natural}(K)$ can be obtained from the following equivalent characterization, which uses a cobordism from $K$ to the unknot rather than the other way around.

Lemma 5.1. Let $\sigma: \mathscr{R} \rightarrow \mathcal{S}$ be a base change with $\sigma\left(T_{0}\right)=\sigma\left(T_{1}\right)$. For a classical $k$ not $K$, let $\Sigma$ be an oriented immersed cobordism from $K$ to the unknot $U_{1}$. Let

$$
\mathscr{I} \subset I^{\natural}\left(U_{1} ; \Gamma_{\sigma}\right) \cong \mathcal{S}
$$

be the image of $I^{\natural}\left(\Sigma ; \Gamma_{\sigma}\right)$, regarded as an ideal in $\mathcal{S}$ via the isomorphism. Then

$$
z_{\sigma}^{\natural}(K)=\sigma(P)^{-\gamma(\Sigma)} \sigma(L)^{-\varepsilon(\Sigma)} \mathscr{I},
$$

as fractional ideals for $\mathcal{S}$, where $\gamma$ and $\varepsilon$ are the genus and number of positive double points.

Proof. Let $S$ be a cobordism from $U_{1}$ to $K$. To abbreviate our notation, we identify the reduced homology of $U_{1}$ with $\delta$ and we write $N$ for the module $I^{\natural}\left(K ; \Gamma_{\sigma}\right) /$ torsion. Let $i(S)$ and $i(\Sigma)$ denote the maps induced by these cobordisms modulo torsion:

$$
\begin{aligned}
& i(S): \mathcal{S} \rightarrow M \\
& i(\Sigma): M \rightarrow \mathcal{S} .
\end{aligned}
$$

We regard $M$ itself as a fractional ideal in $M \otimes$ Frac $\delta$. With that in mind, we have previously defined the fractional ideal $\zeta_{\sigma}^{\natural}(K)$ as

$$
\zeta_{\sigma}^{\natural}(K)=\{c \in \operatorname{Frac}(\delta) \mid c i(S)(1) \in M\} .
$$


The map $i(S)$ is an isomorphism from $M$ to its image $\mathscr{I} \subset \mathcal{S}$, so we can write

$$
\zeta_{\sigma}^{\natural}(K)=\{c \in \operatorname{Frac}(S) \mid c i(\Sigma) i(S)(1) \in \mathscr{I}\} .
$$

The composite cobordism $S \cup \sum$ from $U_{1}$ to $U_{1}$ gives rise to the map

$$
i(\Sigma) i(S)=\sigma(P)^{\gamma(S \cup \Sigma)} \sigma(L)^{\varepsilon(S \cup \Sigma)} .
$$

So

$$
\zeta_{\sigma}^{\natural}(K)=\sigma(P)^{-\gamma(S \cup \Sigma)} \sigma(L)^{-\varepsilon(S \cup \Sigma)} \mathscr{I} .
$$

By definition of $z_{\sigma}^{\natural}$,

$$
\begin{aligned}
z_{\sigma}^{\natural} & =\sigma(P)^{\gamma(S)} \sigma(L)^{\varepsilon(S)} \zeta_{\sigma}^{\natural}(K) \\
& =\sigma(P)^{-\gamma(\Sigma)} \sigma(L)^{-\varepsilon(\Sigma)} \mathscr{J}
\end{aligned}
$$

as the lemma claimed.

\section{Concordance homomorphisms}

We return to classical knots $K \subset S^{3}$ and Proposition 3.13. We can use this result to define homomorphisms from the knot concordance group, in the spirit of Rasmussen's $s$-invariant [15] or the $\tau$-invariant of Ozsvath and Szabo [10].

We consider a base change $\sigma: \mathscr{R} \rightarrow \mathcal{S}$, where $\mathcal{S}$ is a valuation ring. That is, writing $\operatorname{Frac}(\mathcal{S})$ for the field of fractions, we have a surjective homomorphism of groups,

$$
\text { ord }: \operatorname{Frac}(\delta)^{\times} \rightarrow G
$$

where $G$ is a totally ordered group, written additively, and

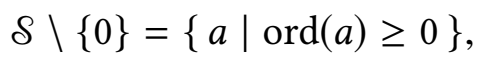

(following the conventions of [4]). Every finitely-generated fractional ideal of $\delta$ is principal, and ord gives rise to a bijection

$$
\text { ord : }\{\text { non-zero principal fractional ideals }\} \rightarrow G
$$

with $\operatorname{ord}(I) \operatorname{ord}(J)=\operatorname{ord}(I)+\operatorname{ord}(J)$, and $\operatorname{ord}(I) \geq \operatorname{ord}(J)$ if and only if $I \subset J$. (In this way, the valuation group $G$, the total order on $G$, and the homomorphism ord are all determined up to equivalence by the structure of $\mathcal{S}$ alone.) We suppose as 
always that $\sigma(P)$ and $\sigma(L)$ are non-zero, and in this case we have distinguished elements of the valuation group,

$$
\begin{aligned}
\pi & =\operatorname{ord}(\sigma(P)) \\
\lambda & =\operatorname{ord}(\sigma(L)) .
\end{aligned}
$$

Suppose now that $\sigma\left(T_{0}\right)=\sigma\left(T_{1}\right)$, so that the reduced group $I^{\natural}\left(K ; \Gamma_{\sigma}\right)$ is defined. Consider the fractional ideal $z_{\sigma}^{\natural}(K)$ associated to a knot $K$ and the basechange $\sigma$, in the reduced version. Since this ideal is finitely generated, it is principal. It is also a concordance invariant of $K$, so we make the following definition:

Definition 5.2. Let $\sigma: \mathscr{R} \rightarrow \mathcal{S}$ be a base-change with $\sigma\left(T_{0}\right)=\sigma\left(T_{1}\right)$. Suppose $\mathcal{S}$ is a valuation ring with valuation group $G$. Then we define a map

$$
f_{\sigma}: \text { Conc } \rightarrow G
$$

where Conc is the knot concordance group, by $f_{\sigma}(K)=\operatorname{ord}\left(z_{\sigma}^{\natural}(K)\right)$.

Proposition 5.3. The map $f_{\sigma}$ is a group homomorphism.

Proof. It is only necessary to prove that $f_{\sigma}\left(K_{1} \# K_{2}\right)=f_{\sigma}\left(K_{1}\right)+f_{\sigma}\left(K_{2}\right)$, which is equivalent to an equality of principal fractional ideals,

$$
z_{\sigma}^{\natural}\left(K_{1} \# K_{2}\right)=z_{\sigma}^{\natural}\left(K_{1}\right) z_{\sigma}^{\natural}\left(K_{2}\right) .
$$

For a valuation ring such as $\delta$, just as for a principal ideal domain, every finitelygenerated submodule of a finitely-generated free module is free, and every finitely-presented module is a direct sum of a free module and torsion modules of the form $S / A$, where $A$ is a principal ideal. In particular, every finitely-presented module has a free resolution of length 1 by finite-rank modules, and the Künneth theorem for a tensor product of differential modules holds in the same form as for principal ideal domains: there is a natural short exact sequence as in [9, Proposition 2.4], and the sequence splits.

Let $I^{\natural}\left(K ; \Gamma_{\sigma}\right)^{\prime}$ denote again the quotient of $I^{\natural}\left(K ; \Gamma_{\sigma}\right)$ by its torsion submodule. The fact that the sequence [9, equation (24)] splits implies that the natural map

$$
I^{\natural}\left(K_{1} ; \Gamma_{\sigma}\right)^{\prime} \otimes_{\mathcal{S}} I^{\natural}\left(K_{2} ; \Gamma_{\sigma}\right)^{\prime} \longrightarrow I^{\natural}\left(K_{1} \# K_{2} ; \Gamma_{\sigma}\right)^{\prime}
$$

is an isomorphism of free rank-1 modules. For $i=1,2$, let $S_{i}$ be a based cobordism from the unknot to $K_{i}$. Let $S$ be the cobordism from the unknot to $K_{1} \# K_{2}$ obtained 
by summing along the base-point arc. These three cobordisms give rise to maps $\phi_{1}, \phi_{2}$ and $\phi$ on reduced instanton homology:

$$
\begin{aligned}
\phi_{i} & : \mathcal{S} \rightarrow I^{\natural}\left(K_{i} ; \Gamma_{\sigma}\right)^{\prime} \cong \mathcal{S} \\
\phi & : \mathcal{S} \rightarrow I^{\natural}\left(K_{1} \# K_{2} ; \Gamma_{\sigma}\right)^{\prime} \cong \mathcal{S} \otimes_{\mathcal{S}} \mathcal{S} \cong \mathcal{S} .
\end{aligned}
$$

The three maps are each multiplication by elements $\zeta_{1}, \zeta_{2}$ and $\zeta$, which are welldefined up to units. The naturality of the isomorphism (16) with respect to cobordisms implies that $\zeta \sim \zeta_{1} \zeta_{2}$.

The definition of $z_{\sigma}^{\natural}$ means that

$$
z_{\sigma}^{\natural}\left(K_{i}\right)=\left\langle P^{\gamma\left(S_{i}\right)} \zeta_{i}\right\rangle
$$

for $i=1,2$ and

$$
z_{\sigma}^{\natural}(K)=\left\langle P^{\gamma(S)} \zeta\right\rangle .
$$

The genus is additive and $\zeta \sim \zeta_{1} \zeta_{2}$, so the desired equality of principal ideals (15) follows.

Proposition 5.4. Let $S$ be a connected, oriented, normally immersed cobordism from $K_{0}$ to $K_{1}$. Let $\gamma(S)$ be its genus and $\varepsilon(S)$ the number of positive double points. Let $\sigma$ be a base change as in Definition 5.2. Then

$$
\gamma(S) \pi+\varepsilon(S) \lambda \geq f_{\sigma}\left(K_{1}\right)-f_{\sigma}\left(K_{0}\right) .
$$

In particular, for an oriented, immersed cobordism from the unknot $U_{1}$ to $K$ (or equivalently an oriented immersed surface in the four-ball), we have

$$
\gamma(S) \pi+\varepsilon(S) \lambda \geq f_{\sigma}(K)
$$

In the case of embedded surfaces, we deduce that the slice genus $g_{s}(K)$ satisfies

$$
g_{s}(K) \geq \frac{1}{\pi} f_{\sigma}(K)
$$

Proof. This is a consequence of Proposition 3.13 and the definitions.

If the base-change $\sigma$ has $\sigma\left(T_{0}\right)=1$ in addition to having target ring $\mathcal{S}$ a valuation ring, then we can adapt the theorem on non-orientable surfaces, Theorem 4.1. Parallel to the proposition above, we then have: 
Proposition 5.5. Let $S$ be a possibly non-orientable, connected surface normally immersed in $B^{4}$ with boundary $K \subset S^{3}$. Let $\sigma$ be a base change as in Definition 5.2, and suppose in addition that $\sigma\left(T_{0}\right)=1$. Let $\eta(S) \in \mathbb{Z}$ be defined again by (10). Then we have

$$
\eta(S) \geq \frac{1}{\pi} f_{\sigma}(K)
$$

In the case of an embedded surface $S$, the inequality of the last proposition can be written as

$$
\frac{1}{2}\left(b_{1}(S)-\frac{1}{2} v(S)\right) \geq \frac{1}{\pi} f_{\sigma}(K) .
$$

As stated in the introduction, this inequality has the same form as the inequality for non-orientable surfaces in [14], with $f_{\sigma}(K)$ replacing the invariant $\Upsilon_{K}(1)$ from [14] (and a different normalization). As in [14], one can exploit the GordonLitherland inequality to derive an inequality that does not involve the degree of the normal bundle, $v(S)$, but instead involves the signature of the knot:

$$
b_{1}(S) \geq \frac{1}{\pi} f_{\sigma}(K)+\frac{1}{2} \operatorname{signature}(K) .
$$

Substantial lower bounds for the betti number of a non-orientable surface bounding a given knot were first obtained by Batson [5], who also observed that the torus knots $T_{2 k, 2 k-1}$ bounds a non-orientable surfaces $S_{k}$ whose betti numbers have linear growth in $k$. The torus knot $K=T_{2 k, 2 k-1}$ has signature $-2 k^{2}+2$, so the above inequality implies

$$
\frac{1}{\pi} f_{\sigma}(K) \leq k^{2}+O(k)
$$

On the other hand, the slice genus of this torus knot is $(k-1)(2 k-1)$, which is $2 k^{2}$ to leading order. So the inequality for the (usual orientable) slice-genus (17) in these cases fails to be sharp, by a factor of 2 for large $k$, for base-changes with $\sigma\left(T_{0}\right)=1$.

\section{Examples}

We now illustrate the workings of the concordance homomorphisms $f_{\sigma}$, for suitable base-changes $\sigma: \mathscr{R} \rightarrow \mathcal{S}$ to valuation rings $\mathcal{S}$. 
Example A. Let $\mathbb{K}$ be any field extension of $\mathbb{F}_{2}$, and let $\operatorname{Frac}(\mathcal{S})$ be the Novikov field of formal sums

$$
\operatorname{Frac}(\mathcal{S})=\left\{\sum_{\alpha \in \mathbb{R}} k_{\alpha} x^{\alpha} \mid k_{\alpha} \in \mathbb{K} \text {, and } \forall C \in \mathbb{R},\left\{\alpha \mid \alpha<C, k_{\alpha} \neq 0\right\} \text { is finite }\right\}
$$

The valuation group $G$ is $\mathbb{R}$, and for convenience we normalize the valuation by declaring that

$$
\operatorname{ord}(x)=1 / 4
$$

so that

$$
\operatorname{ord}\left(\sum_{\alpha \in \mathbb{R}} k_{\alpha} x^{\alpha}\right)=\frac{1}{4} \min \left\{\alpha \mid k_{\alpha} \neq 0\right\} .
$$

The ring $\delta$ comprises as always the elements with ord $\geq 0$ together with 0 . We take $\sigma: \mathscr{R} \rightarrow \mathcal{S}$ to have the form

$$
\sigma\left(T_{i}\right)=1+p_{i}(x), \quad i=1,2,3
$$

where the Novikov series $p_{i}(x)$ have ord $\left(p_{i}(x)\right)>0$ (and $\sigma\left(T_{0}\right)=\sigma\left(T_{1}\right)$, as required for the reduced theory). For any such choices, we have a homomorphism,

$$
f_{\sigma}: \text { Conc } \rightarrow \mathbb{R}
$$

As a first case, we may take, for example,

$$
p_{i}(x)=q_{i} x
$$

where $q_{1}, q_{2}, q_{3} \in \mathbb{K}$ are algebraically independent transcendental elements over $\mathbb{F}_{2}$. In this case we calculate

$$
\sigma(P)=\left(q_{2}^{2} q_{3}^{2}+q_{3}^{2} q_{1}^{2}+q_{1}^{2} q_{2}^{2}\right) x^{4}+\text { higher order in } x
$$

and

$$
\sigma(L)=\left(q_{1}^{4}+q_{2}^{2} q_{3}^{2}+q_{3}^{2} q_{1}^{2}+q_{1}^{2} q_{2}^{2}\right) x^{4}+\text { higher order in } x .
$$

Our convention (18) means that both of these have order 1 , so $\pi=\lambda=1$, and the inequality for an immersed, oriented cobordism $S$ in Proposition 5.4 is

$$
\gamma(S)+\varepsilon(S) \geq f_{\sigma}\left(K_{1}\right)-f_{\sigma}\left(K_{0}\right) .
$$


Example B. As a modification of the previous example, we may fix $r \in[0,1]$ and set

$$
p_{1}(x)=q_{1} x^{r}
$$

and

$$
p_{2}(x)=p_{3}(x)=q_{2} x
$$

In this case

$$
\sigma(P)=q_{2}^{4} q_{3}^{2} x^{4}+\text { higher order in } x
$$

and

$$
\sigma(L)=q_{1}^{4} x^{4 r}+\text { higher order in } x .
$$

So $\pi=1$ and $\lambda=r$. We write the corresponding concordance homomorphism as $f_{r}:$ Conc $\rightarrow \mathbb{R}$. The inequality for immersed cobordisms becomes

$$
\gamma(S)+r \varepsilon(S) \geq f_{r}\left(K_{1}\right)-f_{r}\left(K_{0}\right) .
$$

Example C. As a sort of limit of the previous examples, let $\operatorname{Frac}(\delta)$ be the field of formal Laurent series in $x$ whose coefficients are formal Laurent series in $y$ :

$$
\operatorname{Frac}(\delta)=\mathbb{F}_{2}((y))((x))
$$

Let $G$ be the ordered group $\mathbb{R} \times \mathbb{R}$, lexicographically ordered with the first entry most significant, and define the valuation, ord $: \operatorname{Frac}(\mathcal{S}) \rightarrow G$, by

$$
\operatorname{ord}\left(x^{a} y^{b}\right)=\frac{1}{4}(a, b) .
$$

The valuation ring $\delta$ consists of those elements of $\mathbb{F}_{2}((y))[[x]]$ whose monomials $x^{a} y^{b}$ either have $a>0$ or have $a=0$ and $b \geq 0$. Define $\sigma: \mathscr{R} \rightarrow \mathcal{S}$ by

$$
\begin{aligned}
& \sigma\left(T_{1}\right)=1+y \\
& \sigma\left(T_{2}\right)=1+x
\end{aligned}
$$

with $\sigma\left(T_{0}\right)=\sigma\left(T_{1}\right)$ and $\sigma\left(T_{3}\right)=\sigma\left(T_{2}\right)$. We calculate

$$
\sigma(P)=x^{4}+\text { higher order in }(x, y)
$$

and

$$
\sigma(L)=y^{4}+\text { higher order in }(x, y)
$$


So

$$
\begin{aligned}
& \operatorname{ord}(P)=(1,0) \\
& \operatorname{ord}(L)=(0,1) .
\end{aligned}
$$

This example gives rise (a priori) to a homomorphism

$$
f_{*}: \text { Conc } \rightarrow \mathbb{R} \times \mathbb{R}
$$

with values in $\left(\frac{1}{4} \mathbb{Z}\right) \times\left(\frac{1}{4} \mathbb{Z}\right)$, lexicographically ordered. However, since any two knots cobound an immersed annulus (genus 0 ), we have a bound

$$
f_{*}(K) \leq\left(0, N_{K}\right)
$$

for every knot $K$. So in fact the first component of $f_{*}(K)$ is always zero and we have

$$
\begin{aligned}
f_{*}: \text { Conc } \rightarrow\{0\} & \times\left(\frac{1}{4} \mathbb{Z}\right) \\
& \cong \frac{1}{4} \mathbb{Z} .
\end{aligned}
$$

This concordance homomorphism satisfies

$$
\varepsilon(S) \geq f_{*}\left(K_{1}\right)-f_{*}\left(K_{0}\right)
$$

whenever $S$ is a normally immersed cobordism of genus 0 , from $K_{0}$ to $K_{1}$. Prima facie, it says nothing about normally immersed surfaces of positive genus and does not bound the slice genus of a knot. As stated in the introduction, it would be very interesting to know if there really is a knot $K$ for which $f_{*}(K)$ is larger than the slice genus.

Example $C^{\prime}$. One can simplify Example $\mathrm{C}$ while retaining its features by passing to the ring $S_{1}=\mathbb{F}_{2}[[y]]$ by setting $x=0$. That is, we define:

$$
\begin{aligned}
& \sigma_{1}\left(T_{0}\right)=\sigma_{1}\left(T_{1}\right)=1+y, \\
& \sigma_{1}\left(T_{2}\right)=\sigma_{1}\left(T_{3}\right)=1 .
\end{aligned}
$$

We still have $\sigma_{1}(L)=y^{4}$ to leading order, but $\sigma_{1}(P)=0$. A normally immersed cobordism $S$ of positive genus, between classical knots, now gives the zero map on instanton homology groups, while immersed cobordisms of genus 0 give homomorphisms of rank 1 , between modules of rank 1 over $\delta_{1}$. The concordance homomorphism $f_{*}$ in this example satisfies the same inequality (20) as above. The set-up here is very close to that of [8], though we are now working in characteristic 2 rather than characteristic 0 . In [8], the counterpart of $\sigma_{1}(P)$ was non-zero but divisible by 2 . 
It is interesting to note that, since $\sigma_{1}(P)=\sigma_{1}(Q)=0$ in this example, the resulting spectral sequence has $E_{2}$ page the undeformed reduced Khovanov homology (tensored by $\mathcal{S}_{1}$ ), so we have

$$
\tilde{K h}(\bar{K}) \otimes \mathbb{F}_{2}((y)) \Longrightarrow I^{\natural}\left(K ; \Gamma_{\sigma_{1}}\right) .
$$

Example D. If the base-change $\sigma$ satisfies $\sigma\left(T_{0}\right)=\sigma\left(T_{1}\right)=1$, then the resulting concordance homomorphism $f_{\sigma}:$ Conc $\rightarrow G$ provides a bound for the topology of non-orientable immersed cobordisms, as a consequence of Theorem 4.1 and Proposition 5.5. As a particular case, let $\delta$ be the ring of formal power series $\mathbb{F}_{2}[[x]]$, and define $\sigma$ by

$$
\begin{array}{r}
\sigma\left(T_{0}\right)=\sigma\left(T_{1}\right)=1 \\
\sigma\left(T_{2}\right)=\sigma\left(T_{3}\right)=1+x .
\end{array}
$$

In this example $P$ and $L$ are equal, and $\pi=\lambda=1$ if we set $\operatorname{ord}(x)=1 / 4$ as before. This gives rise to a concordance homomorphism $f_{\sigma}:$ Conc $\rightarrow \mathbb{Z}$ with the property that if $S$ is a possibly non-orientable cobordism from $K_{0}$ to $K_{1}$, then

$$
\eta(S) \geq f_{\sigma}\left(K_{1}\right)-f_{\sigma}\left(K_{0}\right)
$$

where $\eta$ is as in equation (10).

Example E. The following is a hypothetical example, to illustrate the potential workings of the concordance invariants $f_{\sigma}$. Let $\sigma: \mathscr{R} \rightarrow \mathcal{S}$ be the quotient map by the ideal $\left\langle T_{1}-T_{0}\right\rangle$ in $\mathscr{R}$. Thus $\delta$ is the ring $\mathcal{S}_{\mathrm{BN}}$ of the introduction, a ring of Laurent series in $T_{1}, T_{2}, T_{3}$. This is the largest quotient for which the reduced homology $I^{\natural}\left(K ; \Gamma_{\sigma}\right)$ is defined. We write the images of $P$ and $L$ in $\delta$ simply as $P$ and $L$ again. Suppose that the chain complex that computes $I^{\natural}\left(K ; \Gamma_{\sigma}\right)$ is chainhomotopy equivalent to

$$
\begin{gathered}
\mathcal{S}=C_{0} \stackrel{\partial}{\longrightarrow} C_{1}=\mathcal{S} \oplus, \\
1 \mapsto\left(L^{3}, P\right) .
\end{gathered}
$$

The homology is then generated by 2 elements $[u]$, [v], with chain representatives in $C_{1}$, satisfying

$$
L^{3}[u]=P[v] .
$$

Suppose there is a genus- 1 embedded cobordism $S$ from the unknot to $K$, and that the resulting map of reduced homologies on the chain level is

$$
\begin{gathered}
\mathcal{S} \longrightarrow C_{1} \\
1 \mapsto u .
\end{gathered}
$$


The corresponding fractional ideal $\zeta_{\sigma}^{\natural}(S)$ is then generated by 1 and $P^{-1} L^{3}$. The concordance-invariant ideal $z_{\sigma}^{\natural}(K)$ is $\left\langle P, L^{3}\right\rangle$. In this hypothetical case, Proposition 3.12 would allow that $K$ bounds an immersed disk in the 4-ball with three positive double points, but no fewer. The concordance homomorphism $f_{r}:$ Conc $\rightarrow \mathbb{R}$ from (19) can then be calculated using the universal coefficient theorem. It will take the values,

$$
f_{r}(K)= \begin{cases}3 r, & 0<r \leq 1 / 3 \\ 1, & 1 / 3 \leq r<1\end{cases}
$$

The constraint (19) on the genus and the number of positive double-points coming from $f_{r}$ will therefore be

$$
\gamma+r \varepsilon \geq \begin{cases}3 r, & 0 \leq r \leq 1 / 3 \\ 1, & 1 / 3 \leq r \leq 1\end{cases}
$$

Taken together over all $r$ in $[0,1]$, these constraints are equivalent to the constraint coming from $z_{\sigma}^{\natural}$. That is, if $S$ is embedded then it must have genus 1 at least, while if $S$ is immersed with genus 0 then it must have at least three positive double points. The concordance homomorphism $f_{*}(K)$ in such an example would be 3 .

Of course, the exponent 3 in this hypothetical example is arbitrary. We shall show in section 8 that the positive trefoil behaves in this way, but with the less interesting exponent 1 in place of 3 . (The positive trefoil bounds both an embedded surface of genus 1 and a disk with one positive double point.)

\section{Unknotting number and other properties}

\section{Unknotting number}

As in $[1,2,3]$, one can exploit the torsion in $I^{\sharp}\left(K ; \Gamma_{\sigma}\right)$ or $I^{\natural}\left(K ; \Gamma_{\sigma}\right)$ instead of the torsion-free quotient, to obtain bounds on the unknotting number of $K$, or the crossing-change distance between knots. Suppose $K_{0}$ can be obtained from $K_{1}$ by $n$ crossing-changes, and let $S_{10}$ be the corresponding immersed cylindrical cobordism from $K_{1}$ to $K_{0}$. The composite cobordism $S$ from $K_{1}$ to $K_{1}$, formed as the union of $S_{10}$ and its mirror image, has $2 n$ double points which come in mirror pairs. This cobordism can be obtained from the trivial cylinder by doing $n$ finger-moves, and intermediate isotopies. It follows that

$$
I^{\sharp}(S ; \Gamma)=L^{n} .
$$


In particular then, the map

$$
L^{n}: I^{\sharp}\left(K_{1} ; \Gamma\right) \rightarrow I^{\sharp}\left(K_{1} ; \Gamma\right)
$$

factors through $I^{\sharp}\left(K_{0} ; \Gamma\right)$. To draw a concrete consequence from this, note that $I^{\sharp}\left(K_{0} ; \Gamma\right)$ and $I^{\sharp}\left(K_{1} ; \Gamma\right)$ both have rank 2 , from which it follows that any torsion element $w$ in the image of any full-rank homomorphism $\phi: I^{\sharp}\left(K_{0} ; \Gamma\right) \rightarrow I^{\sharp}\left(K_{1} ; \Gamma\right)$ is necessarily of the form $\phi(v)$ for some torsion element in $I^{\sharp}\left(K_{0} ; \Gamma\right)$. So, in (21), the restriction of the multiplication map by $L^{n}$ to the torsion part of $I^{\sharp}\left(K_{1} ; \Gamma\right)$ factors through the torsion part of $I^{\sharp}\left(K_{0} ; \Gamma\right)$.

We can therefore deduce:

Proposition 7.1 (cf. $[1,2,3])$. If $K_{0}$ can be obtained from $K_{1}$ by $n$ crossing changes, and if $H \in \mathscr{R}$ annihilates the torsion in $I^{\sharp}\left(K_{0} ; \Gamma\right)$, then $L^{n} H$ annihilates the torsion in $I^{\sharp}\left(K_{1} ; \Gamma\right)$.

As a special case, taking $K_{0}$ to be the unknot:

Corollary 7.2 (cf. Theorem 1.2 of [1]). If $K$ has unknotting number $n$, then $L^{n}$ annihilates the torsion in $I^{\sharp}(K ; \Gamma)$. In particular, for any knot $K$, the torsion in $I^{\sharp}(K ; \Gamma)$ is annihilated by some power of $L$.

The result can be recast if we apply a base-change $\sigma: \mathscr{R} \rightarrow \mathcal{S}$ to a valuation ring $\mathcal{S}$. Let the torsion submodule of $I^{\sharp}\left(K_{1} ; \Gamma_{\sigma}\right)$ be

$$
\left(\mathcal{S} / I_{1}\right) \oplus\left(\mathcal{S} / I_{2}\right) \oplus \cdots \oplus\left(\mathcal{S} / I_{l}\right)
$$

with $\operatorname{ord}\left(I_{1}\right) \geq \operatorname{ord}\left(I_{2}\right) \geq \cdots \geq \operatorname{ord}\left(I_{l}\right)$ in $\operatorname{Val}(\delta)$. Thus $I_{1}$ is the annihilator of the torsion part. Let $\tau\left(K_{1}\right)=\operatorname{ord}\left(I_{1}\right)$. Define $\tau\left(K_{0}\right)$ similarly for the other knot. Then the above factorization requires (as a special case),

$$
n \lambda \geq \tau\left(K_{1}\right)-\tau\left(K_{0}\right)
$$

where $\lambda$ is the order of $\sigma(L)$ as before. This goes both ways, so the crossingchange distance between the two knots is therefore bounded below by

$$
\min \left\{n: n \lambda \geq\left|\tau\left(K_{1}\right)-\tau\left(K_{0}\right)\right|\right\}
$$

or simply by

$$
\frac{\left|\tau\left(K_{1}\right)-\tau\left(K_{0}\right)\right|}{\lambda}
$$


if $\operatorname{Val}(\delta) \subset \mathbb{R}$. As a special case, with $K_{0}$ the unknot again, the unknotting number of $K$ is bounded below by $\tau(K) / \lambda$, where $\tau(K)$ is the order of the annihilator of the torsion submodule in $I^{\sharp}\left(K ; \Gamma_{\sigma}\right)$.

There is a slight strengthening of Proposition 7.1 and Corollary 7.2. We are free to modify the definition we have made for how to define the map $I^{\sharp}\left(S ; \Gamma_{\sigma}\right)$ when $S$ has double points, by using the modified formula at (5), so defining a modified functor $I_{\xi}^{\sharp}$. Recall that $I^{\sharp}(K ; \Gamma)$ is unchanged, as only the maps induced by immersed surfaces are modified. In this case, the formula for $L$ must be replaced by

$$
L_{\xi}=\xi P+T_{0}^{2}+T_{0}^{-2} .
$$

(See [9, Proposition 4.9].) The conclusions of Proposition 7.1 and Corollary 7.2 continue to hold with $L_{\xi}$ in place of $L$. That is, for example, if $K$ has unknotting number $n$, then $L_{\xi}^{n}$ annihilates the torsion in $I^{\sharp}(K ; \Gamma)$. Here $\xi$ is arbitrary, so we can take our ground ring to be $\mathscr{R}[\xi]$ where $\xi$ is an indeterminate. The statement that $L_{\xi}^{n}$ annihilates the torsion is then equivalent to the statement that $L^{a} P^{b}$ annihilates the torsion, for all $a, b$ with $a+b=n$. We record this as a variant of Corollary 7.2.

Corollary 7.3. If $K$ has unknotting number $n$, then the torsion in $I^{\sharp}(K ; \Gamma)$ is annihilated by the ideal $\langle L, P\rangle^{n}$.

If we pass to a valuation ring, then ord $(L)$ will in general be less than or equal to $\operatorname{ord}(P)$, in which case the ideal $\langle L, P\rangle$ is simply $\langle L\rangle$, so the variant is equivalent to the original in this case.

\section{Ribbon concordance}

Related to the above arguments involving the unknotting number, the functoriality of both Khovanov homology and Heegaard knot Floer homology has been used by Zemke [18] and Levine-Zemke [19], to obtain constraints on the existence of a ribbon concordance from a knot $K_{0}$ to a knot $K_{1}$. (A ribbon concordance is an embedded annulus in $[0,1] \times S^{3}$ such that the first coordinate function is Morse and has no index- 2 critical points.) Since the argument is quite formal, it adapts to the case of instanton homology without essential change:

Theorem $7.4(c f .[18,19])$. If $S$ is a ribbon concordance from $K_{0}$ to $K_{1}$, then the resulting map $I^{\sharp}(S ; \Gamma)$ is injective. 
As in [18], the proof proceeds by considering the composite of $S$ with its mirror image, which is a cobordism from $K_{0}$ to $K_{0}$ which can be repeatedly be simplified by neck-cutting.

\section{Calculation for the trefoil}

\section{Statement of the result}

In this section we take $K$ to be the trefoil. We work again with the reduced homology $I^{\natural}\left(K ; \Gamma_{\sigma}\right)$, and we take the base change $\sigma: \mathscr{R} \rightarrow \mathcal{S}$ to the largest quotient of $\mathscr{R}$ for which the reduced theory is defined. As in the introduction and Example $\mathrm{E}$ from Section 5, this ring $\delta$ is the Laurent series ring $\delta_{\mathrm{BN}}$ in three variables $T_{i}, i=1,2,3$, and $\sigma_{b n}$ is the quotient map by the ideal generated by $T_{0}-$ $T_{1}$. The local system $\Gamma_{\sigma_{b n}}$ coincides with the system named $\Gamma_{\mathrm{BN}}$ in the introduction. We again simply write $P$ and $L$ for the Laurent polynomials which are really $\sigma_{b n}(P)$ and $\sigma_{b n}(L)$. In particular then,

$$
L=T_{1}^{2}+T_{1}^{-2}+P
$$

We write $z_{\mathrm{BN}}^{\natural}(K)$ for $z_{\sigma_{b n}}^{\natural}(K)$ in this case, and we will compute the fractional ideal $z_{\mathrm{BN}}^{\natural}(K) \subset \operatorname{Frac}\left(\mathcal{S}_{\mathrm{BN}}\right)$ for the trefoil.

Proposition 8.1. For the right-handed trefoil $K_{2,3}$, the complex of free $S_{\mathrm{BN}}$-modules that computes $I^{\natural}\left(K_{2,3} ; \Gamma_{\mathrm{BN}}\right)$ is chain-homotopy equivalent to the complex

$$
\delta_{\mathrm{BN}} \stackrel{\partial}{\rightarrow} \delta_{\mathrm{BN}} \oplus \delta_{\mathrm{BN}}
$$

where $\partial(1)=(L, P)$. In particular, $I^{\natural}\left(K_{2,3} ; \Gamma_{\mathrm{BN}}\right)$ has a presentation with two generators and one relation, $L e_{1}+P e_{2}=0$, which means that it isomorphic to the ideal $J=\langle P, L\rangle$ as an $S_{\mathrm{BN}}$-module. Furthermore, the fractional ideal $z_{\mathrm{BN}}^{\natural}\left(K_{2,3}\right)$ coincides with $J$.

Remark. After the preparation of earlier drafts of this paper, it became apparent that it is possible to prove this result with no reference to "instantons" beyond what is already built into the formal properties of $I^{\natural}(K ; \Gamma)$. Nevertheless, in the proof we give here (in particular in Lemma 8.2 below), we obtain some explicit information at the chain level by considering instanton moduli spaces, so making contact with the constructions that underlie the definitions. The authors therefore decided to retain this version of the calculation. 


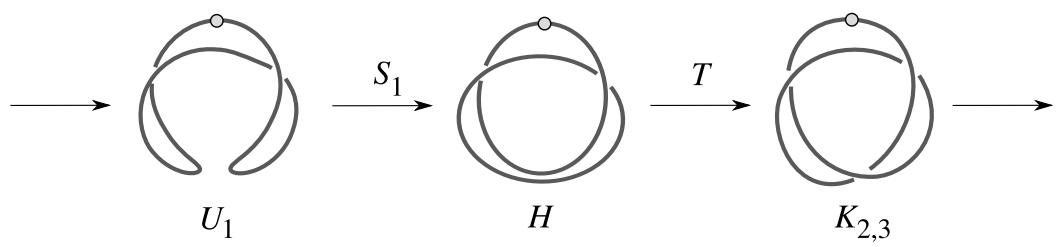

Figure 2: The skein triangle for the positive trefoil $K_{2,3}$, the Hopf link $H$, and the unknot $U_{1}$.

\section{The skein triangle and the Hopf link}

The first step in the proof of Proposition 8.1 is to use the skein exact triangle illustrated in Figure 2. The figure shows the right-handed trefoil together with the result of smoothing one of the three crossings in two different ways. Because we are using reduced instanton homology, we require a base-point on each link, where the bigon is introduced. The location of the base-point is marked by a dot in the figure.

The skein sequence

$$
\cdots \rightarrow U_{1} \rightarrow H \rightarrow K_{2,3} \rightarrow U_{1} \rightarrow \cdots
$$

leads to a long exact sequence of instanton homology groups. Because $I^{\natural}\left(U_{1} ; \mathcal{S}_{\mathrm{BN}}\right)$ is free of rank 1 and the instanton homologies of $H$ and $K_{2,3}$ have rank 2 and 1 respectively, the long exact sequence must break into a short exact sequence,

$$
0 \rightarrow I^{\natural}\left(U_{1} ; \mathcal{S}_{\mathrm{BN}}\right) \stackrel{n}{\rightarrow} I^{\natural}\left(H ; \mathcal{S}_{\mathrm{BN}}\right) \stackrel{k}{\rightarrow} I^{\natural}\left(K_{2,3} ; \mathcal{S}_{\mathrm{BN}}\right) \rightarrow 0
$$

At the chain level, the skein sequence tells us that the corresponding complexes $C^{\natural}\left(U_{1}\right), C^{\natural}(H)$ and $C^{\natural}\left(K_{2,3}\right)$ are related in such a way that $C^{\natural}\left(K_{2,3}\right)$ is chainhomotopy equivalent to the mapping cone of the chain map arising from the cobordism

$$
U_{1} \stackrel{S_{1}}{\rightarrow} H
$$

This cobordism $S_{1} \subset[0,1] \times S^{3}$ is a pair of co-pants, but not with the standard embedding. To calculate the complex for the trefoil, up to chain homotopy, we shall calculate the map arising from the cobordism $S_{1}$ at the chain level.

Before proceeding, we note for later use that we may consider the skein triangle obtained from the smoothings of a crossing on the Hopf link to obtain the 


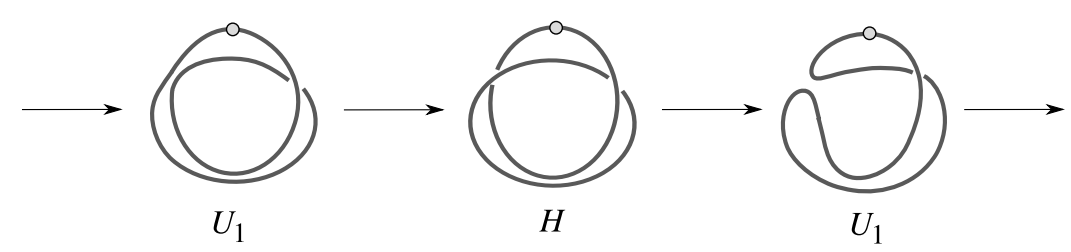

Figure 3: The skein triangle for the Hopf link $H$, and two copies of the unknot $U_{1}$.

sequence in Figure 3. Similar consideration of ranks shows that this gives rise to a short exact sequence,

$$
0 \rightarrow I^{\natural}\left(U_{1} ; \delta_{\mathrm{BN}}\right) \stackrel{i}{\rightarrow} I^{\natural}\left(H ; S_{\mathrm{BN}}\right) \stackrel{p}{\rightarrow} I^{\natural}\left(U_{1} ; \delta_{\mathrm{BN}}\right) \rightarrow 0
$$

Let us write $R^{\natural}(K)$ for the representation variety of marked bifold connections on $K^{\natural}$, where $K^{\natural}$ is obtained from $K$ by adding the bigon [9, Figure 1]. After orienting $K$ near the base-point, let $\mathrm{m}_{0}$ be any representative of the oriented meridian at the base-point, as an element of $\pi_{1}\left(S^{3} \backslash K\right)$. Let $\mathbf{i}$ be the element $\operatorname{diag}(-i, i)$ in $S U(2)$. We can identify $R^{\natural}(K)$ with the space of representations of the link complement,

$$
\rho: \pi_{1}\left(S^{3} \backslash K\right) \rightarrow S U(2)
$$

satisfying the constraint that $\rho\left(\mathrm{m}_{0}\right)=\mathbf{i}$ and $\rho(\mathrm{m})$ is conjugate to $\mathbf{i}$ for all other meridians. A representation $\rho$ gives rise to representation of the orbifold fundamental group of the web $K^{\natural}$ by sending the meridians of the edges $e_{1}$ and $e_{2}$ in $[9$, Figure 1] to $\mathbf{j}$ and $\mathbf{k}$.

The representation variety $R^{\natural}\left(U_{1}\right)$ for the unknot, with this description, consists of a single representation $\alpha$, with $\alpha\left(\mathrm{m}_{0}\right)=\mathbf{i}$. The representation variety of the Hopf link $H$ consists of two representations: the fundamental group of the complement is abelian, so a representation that maps $\mathrm{m}_{0}$ to $\mathrm{i}$ maps a meridian of the other component to $\pm \mathbf{i}$. To distinguish consistently between the two cases, given $\beta \in R^{\natural}(H)$, we can orient the two components of the link so that the both oriented meridians map to i. Oriented in this way, the linking number of the Hopf link will be either 1 or -1 . We name the two elements of $R^{\natural}(H)$ as $\beta_{+}$and $\beta_{-}$respectively. See Figure 4.

The critical points $\alpha, \beta_{+}$and $\beta_{-}$can all be seen to be regular. So the corresponding chain complexes are $\delta_{\mathrm{BN}}$ and $\delta_{\mathrm{BN}} \oplus \delta_{\mathrm{BN}}$ respectively. Furthermore, there is no differential in the latter case. One can see this either by showing that $\beta_{ \pm}$have the same mod 2 grading in this complex, or by noting that the matrix 


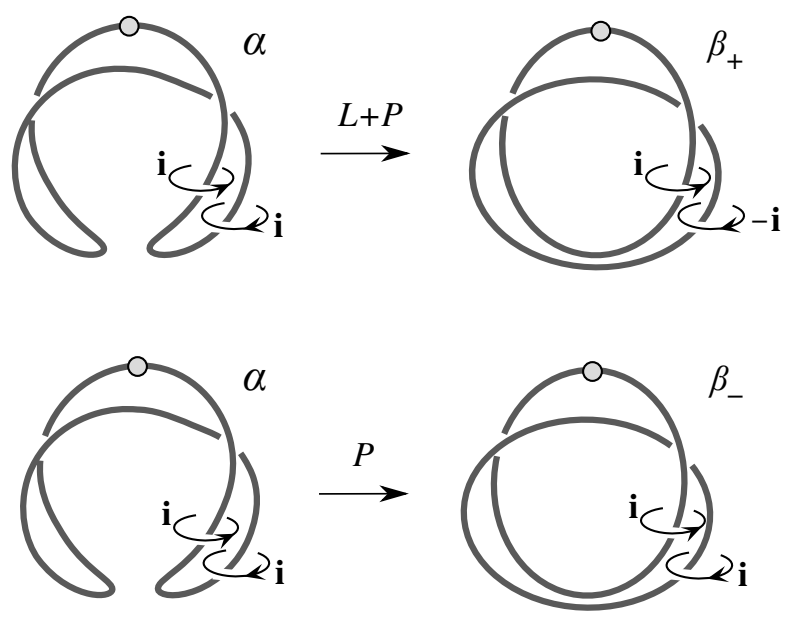

Figure 4: The matrix entries $X_{+}=L+P$ and $X_{-}=P$, from $\alpha$ to $\beta_{+}$and $\beta_{-}$respectively. All loops are based, with the basepoint lying above the plane of the diagram as usual. Not shown are the two arcs of the added bigon, where the monodromies are $\mathbf{j}$ and $\mathbf{k}$.

entry of the differential from $\beta_{+}$to $\beta_{-}$is equal to the matrix entry from $\beta_{-}$to $\beta_{+}$ by symmetry, and noting that a non-zero entry would contradict $d^{2}=0$.

The mapping cone arising from (24) therefore has the form

$$
X=\left(X_{+}, X_{-}\right): \delta_{\mathrm{BN}} \rightarrow \delta_{\mathrm{BN}} \oplus \mathcal{S}_{\mathrm{BN}}
$$

where the two elements $X_{+}, X_{-} \in \delta_{\mathrm{BN}}$ are the matrix entries at the chain level of the map induced by the cobordism $S_{1}$, from $\alpha$ to $\beta_{+}$and $\beta_{-}$. (At the level of homology, this is the map $n$ in (23).) These matrix entries are determined in the next lemma, illustrated in Figure 4.

Lemma 8.2. The elements $X_{+}$and $X_{-}$are $L+P$ and $P$ respectively.

Apart from its last sentence (identifying the fractional ideal), Proposition 8.1 follows from the lemma. Only a change of basis is needed to change the matrix entries in the presentation from $(L+P, P)$ to $(L, P)$.

\section{Proof of the lemma: computing $X_{+}$and $X_{-}$}

We turn to the proof of the lemma. We introduce two additional cobordisms, both from $H$ to $U_{1}$, called $S_{g}$ and $S_{\delta}$ respectively. The cobordism $S_{g}$ is the mirror image of $S_{1}$, so is an embedded pair of pants. The cobordism $S_{\delta}$ will be an 
immersed cobordism with a single double point: it is the union of an embedded cylinder $[0,1] \times S^{1}$ where the $S^{1}$ is the component of $H$ with the base-point, and an embedded disk $D$ whose boundary is the other component of $H$. The disk $D$ meets the cylinder in one point. The composites $S_{1} \circ S_{g}$ and $S_{1} \circ S_{\delta}$ are two cobordisms from $U_{1}$ to $U_{1}$. They are respectively an embedded surface of genus 1 , and an embedded cylinder with one positive double point. We therefore have

$$
\begin{aligned}
& I^{\natural}\left(S_{1} \circ S_{g} ; \Gamma_{\mathrm{BN}}\right)=P \\
& I^{\natural}\left(S_{1} \circ S_{\delta} ; \Gamma_{\mathrm{BN}}\right)=L .
\end{aligned}
$$

By examining the flat connections explicitly, we will see that the cobordism $S_{g}$ maps the generators $\beta_{ \pm}$as follows,

$$
\begin{aligned}
& I^{\natural}\left(S_{g} ; \Gamma_{\mathrm{BN}}\right)\left(\beta_{+}\right)=0 \\
& I^{\natural}\left(S_{g} ; \Gamma_{\mathrm{BN}}\right)\left(\beta_{-}\right)=\alpha
\end{aligned}
$$

while for $S_{\delta}$ we have

$$
\begin{aligned}
& I^{\natural}\left(S_{\delta} ; \Gamma_{\mathrm{BN}}\right)\left(\beta_{+}\right)=\alpha \\
& I^{\natural}\left(S_{\delta} ; \Gamma_{\mathrm{BN}}\right)\left(\beta_{-}\right)=\alpha .
\end{aligned}
$$

From the formulae (27) and (28) and (26), we obtain

$$
I^{\natural}\left(S_{1} ; \Gamma_{\mathrm{BN}}\right)(\alpha)=(L+P) \beta_{+}+P \beta_{-}
$$

which is equivalent to the statement of the lemma.

To complete the proof of the lemma, it remains to prove the formulae (27) and (28) for the cobordisms $S_{g}$ and $S_{\delta}$. Let $H^{\natural}$ and $U_{1}^{\natural}$ be the webs obtained from $H$ and $U_{1}$ by adding bigons near the marked point on each. Let $\hat{S}_{\delta}$ be the proper transform of $S_{\delta}$ after blowing up up at the point of self-intersection. This surface is the disjoint union of an embedded annulus and a disk $D_{\delta}$ whose boundary is the unmarked component of $H$. Let $S_{g}^{\natural}$ and $\hat{S}_{\delta}^{\natural}$ denote the foams obtained from $S_{g}$ and $\hat{S}_{\delta}$ by adding a bigon along arcs joining the marked points. These foams are cobordisms from $H^{\natural}$ to $U_{1}^{\natural}$. Let us write

$$
\begin{aligned}
& W_{g}=\left([0,1] \times S^{3}, S_{g}^{\natural}\right) \\
& W_{\delta}=\left([0,1] \times S^{3} \# \overline{\left.\mathbb{C} \mathbb{P}^{2}, \hat{S}_{\delta}^{\natural}\right)}\right.
\end{aligned}
$$

for the corresponding 4-dimensional bifold cobordisms. The matrix entries of $I^{\natural}\left(S_{g} ; \Gamma_{\mathrm{BN}}\right)$ are defined by counting instantons in zero-dimensional components of the moduli spaces

$$
M\left(\beta_{ \pm} ; W_{g} ; \alpha\right)
$$


on the cobordism $W_{g}$ with cylindrical ends. In the case of the double-point cobordism, the matrix entries of $I^{\natural}\left(S_{\delta} ; \Gamma_{\mathrm{BN}}\right)$ are defined by a similar count in moduli spaces

$$
M\left(\beta_{ \pm} ; W_{\delta} ; \alpha\right) \quad \text { and } \quad M\left(\beta_{ \pm} ; W_{\delta} ; \alpha\right)_{\epsilon}
$$

where $\epsilon$ is the exceptional set of the blow-up. (See the definition at [9, equation (34)].)

There are smooth Klein-four-group covers of these bifolds,

$$
\tilde{W}_{g} \rightarrow W_{g} \quad \text { and } \quad \tilde{W}_{\delta} \rightarrow W_{\delta},
$$

branched over the singular loci $S_{g}^{\natural}$ and $\hat{S}_{\delta}^{\natural}$ respectively. The trivial $S O(3)$ bundles on $\tilde{W}_{g}$ and $\tilde{W}_{\delta}$ descend to flat $S O(3)$ bifold connections $c_{g}$ on $W_{g}$ and $c_{\delta}$ on $W_{\delta}$ respectively. The flat $S O(3)$ bifold connection $c_{g}$ lifts to a unique (flat) $S U(2)$ connection $C_{g}$ on $W_{g}$ and defines an element of the moduli space $M\left(\beta_{-} ; W_{g} ; \alpha\right)$. Similarly $c_{\delta}$ lifts to a flat $S U(2)$ connection $C_{\delta}^{-}$, which defines an element of the moduli space $M\left(\beta_{-} ; W_{\delta} ; \alpha\right)$. On the bifold $W_{\delta}$ however there is a flat line bundle $\xi$ with holonomy -1 on the links of both the exceptional sphere $\epsilon$ and the disk $D_{\delta} \subset \hat{S}_{\delta}$. By twisting $C_{\delta}^{-}$with $\xi$ we obtain an $S U(2)$ connection $C_{\varepsilon}$ in $M\left(\beta_{+} ; W_{\delta} ; \alpha\right)$. Altogether we have three flat connections,

$$
\begin{gathered}
{\left[C_{g}\right] \in M\left(\beta_{-} ; W_{g} ; \alpha\right)} \\
{\left[C_{\delta}^{-}\right] \in M\left(\beta_{-} ; W_{\delta} ; \alpha\right)} \\
{\left[C_{\delta}^{+}\right] \in M\left(\beta_{+} ; W_{g} ; \alpha\right)_{\epsilon} .}
\end{gathered}
$$

The Klein-four-group covers $\tilde{W}_{g}$ and $\tilde{W}_{\delta}$ are two cobordisms from the rational homology sphere $\mathbb{R} \mathbb{P}^{3} \# \mathbb{R} \mathbb{P}^{3}$ to $S^{3}$, and both have $b_{1}=0$ and $b_{2}^{+}=0$. It follows that these three elements (29) have no infinitesimal deformations and are regular points of their respective moduli spaces. Because they are flat, the curvature integrals [9, equation (21)] defining the local systems are trivial, and each of the three connections therefore contributes 1 to the corresponding matrix entry of the map $I^{\natural}\left(S_{g} ; \Gamma_{\mathrm{BN}}\right)$ or $I^{\natural}\left(S_{\delta} ; \Gamma_{\mathrm{BN}}\right)$. There are no other flat connections, and any non-flat connection would belong to a moduli space of strictly positive dimension and would not contribute to the cobordism maps. This completes the verification of the formulae (27) and (28) and so completes the proof of Lemma 8.2.

\section{Identifying the fractional ideal}

We have now completed the proof of the assertion in Proposition 8.1 that $I^{\natural}\left(K_{2,3} ; \Gamma_{S}\right)$ is isomorphic to the ideal $J=\langle P, L\rangle$. To identify the fractional ideal $z_{\mathrm{BN}}^{\natural}\left(K_{2,3}\right)$ we 
need an oriented, immersed cobordism $\Sigma$ from $U_{1}$ to $K_{2,3}$, and for this we can take the composite of the cobordism $T$ from $H$ to $K_{2,3}$ in Figure 2 and the immersed cobordism $S_{\delta}^{\dagger}$ from $U_{1}$ to $H$ obtained by reversing the cobordism $S_{\delta}$ from the lemma. Proposition 8.1 identifies the $I^{\natural}\left(K_{2,3} ; \Gamma_{\mathrm{BN}}\right)$ in terms of generators $e_{1}$ and $e_{2}$ with a relation $L e_{1}+P e_{2}=0$, but the lemma identifies the same group in terms of generators $\left[\beta_{+}\right]$and $\left[\beta_{-}\right]$with the relation $(L+P)\left[\beta_{+}\right]+P\left[\beta_{-}\right]=0$. The change of basis between these two descriptions is

$$
e_{1}=\left[\beta_{+}\right], \quad e_{2}=\left[\beta_{+}\right]+\left[\beta_{-}\right]
$$

Our discussion of $S_{\delta}$ in the proof of the lemma adapts readily to similar case of $S_{\delta}^{\dagger}$ and shows that this cobordism from $U_{1}$ to $H$ gives the map on generators

$$
\alpha \mapsto \beta_{+}+\beta_{-}
$$

The composite cobordism $\Sigma$ from $U_{1}$ to $K_{2,3}$ is therefore

$$
\alpha \mapsto e_{2}
$$

Since $P e_{2}=L e_{1}$ the definition of $\zeta_{\mathrm{BN}}^{\natural}(\Sigma)$ shows that this fractional ideal is generated by 1 and $L^{-1} P$. Accordingly, from the definition (13), the ideal $z_{\mathrm{BN}}^{\natural}\left(K_{2,3}\right)$ is $\langle L, P\rangle$ as Proposition 8.1 claimed. This completes the proof of the proposition.

\section{Left-handed trefoils and the concordance homomorphisms}

The complex that computes the homology of the left-handed trefoil is the dual complex:

Proposition 8.3. For the left-handed trefoil $K_{2,3}^{-}$, the complex of free $\mathcal{S}_{\mathrm{BN}}$-modules that computes $I^{\natural}\left(K_{2,3}^{-} ; \Gamma_{\mathrm{BN}}\right)$ is chain-homotopy equivalent to the complex

$$
S_{\mathrm{BN}} \oplus S_{\mathrm{BN}} \stackrel{\partial}{\rightarrow} S_{\mathrm{BN}}
$$

where $\partial$ has matrix entries $(L, P)$. In particular, $I^{\natural}\left(K_{2,3}^{-} ; \Gamma_{\mathrm{BN}}\right)$ is isomorphic to $\oint_{\mathrm{BN}} \oplus$ $\left(\mathcal{S}_{\mathrm{BN}} / J\right)$ as an $S_{\mathrm{BN}}-$ module, where $J$ is again $\langle L, P\rangle$. Furthermore, the fractional ideal $z_{\mathrm{BN}}^{\natural}\left(K_{2,3}^{-}\right)$is $\langle 1\rangle$.

Proof. Except for the identification of the fractional ideal, this proposition is obtained by dualizing the previous one. If we write $\epsilon_{1}, \epsilon_{2}$ for the basis of $\delta_{\mathrm{BN}} \oplus \mathcal{S}_{\mathrm{BN}}$, then the generator of $S_{\mathrm{BN}}$ summand in $I^{\natural}\left(K_{2,3}^{-} ; \Gamma_{\mathrm{BN}}\right)$ is the element $\tau=P \epsilon_{1}+L \epsilon_{2}$ 
in $\operatorname{ker}(\partial)$. The immersed cobordism $\Sigma$ from $U_{1}$ to $K_{2,3}$ in the proof of the previous proposition gives a cobordism $\Sigma^{\dagger}$ from $K_{2,3}^{-}$to $U_{1}$, and the dual of the previous calculation says that $\Sigma^{\dagger}$ acts as

$$
\begin{aligned}
& \epsilon_{2} \mapsto \alpha \\
& \epsilon_{1} \mapsto 0
\end{aligned}
$$

So $\Sigma^{\dagger}$ maps the generator $\tau$ of $I^{\natural}\left(K_{2,3}^{-} ; \Gamma_{\mathrm{BN}}\right) /$ torsion to $L \alpha$. That is, the image of the map

$$
I^{\natural}\left(\Sigma^{\dagger} ; \Gamma_{\mathrm{BN}}\right): I^{\natural}\left(K_{2,3}^{-} ; \Gamma_{\mathrm{BN}}\right) \rightarrow I^{\natural}\left(U_{1} ; \Gamma_{\mathrm{BN}}\right) \cong \mathcal{S}_{\mathrm{BN}}
$$

is the ideal $\mathscr{F}=L S_{\mathrm{BN}}$. The immersed cobordism $\Sigma^{\dagger}$ has one positive double point, so by the characterization in Lemma 5.1 we have

$$
\begin{aligned}
z_{\mathrm{BN}}^{\natural}\left(K_{2,3}^{-}\right) & =L^{-1} \mathscr{I} \\
& =\langle 1\rangle .
\end{aligned}
$$

This completes the proof of Proposition 8.3.

Having identified the complexes involved, it is a straightforward matter to apply a further change of basis, $\delta_{\mathrm{BN}} \rightarrow \delta$, where $\delta$ is a valuation ring, so that we may compute the real-valued invariants $f_{r}\left(K_{2,3}\right)$ and $f_{r}\left(K_{2,3}^{-}\right)$for the two trefoils, $0 \leq r \leq 1$. (See Example B in section 6.) We obtain

$$
\begin{aligned}
& f_{r}\left(K_{2,3}\right)=r \\
& f_{r}\left(K_{2,3}^{-}\right)=-r .
\end{aligned}
$$

To illustrate the calculation in the case of $K_{2,3}^{-}$, following the line in Proposition 8.3, the complex is now

$$
\mathcal{S} \oplus \stackrel{\partial}{\rightarrow} \mathcal{S}
$$

where

$$
\begin{aligned}
& \partial\left(\epsilon_{1}\right)=u_{1} x^{4 r} \\
& \partial\left(\epsilon_{2}\right)=u_{2} x^{4}
\end{aligned}
$$

where $u_{1}$ and $u_{2}$ are units. The free summand of the homology (the kernel of $\partial$ ) is generated now by $\tau=u_{1}^{-1} u_{2} x^{4-4 r} \epsilon_{1}+\epsilon_{2}$. The map arising from $\Sigma^{\dagger}$ in the proof of Proposition 8.3 maps $\tau$ to $\alpha \in \mathcal{S}$, the generator. Therefore $z_{\mathrm{BN}}^{\natural}\left(K_{2,3}^{-}\right)=L^{-1} \mathcal{S}$ which is the ideal $\left\langle x^{-4 r}\right\rangle$. The invariant $f_{r}\left(K_{2,3}^{-}\right)$is the order of this ideal, which is $-r$, because the order of the ideal $\langle x\rangle$ is $1 / 4$ by convention (18). 


\section{Further calculations}

As mentioned in an earlier remark, it is possible to obtain a description of $I^{\natural}\left(K ; \Gamma_{\mathrm{BN}}\right)$ for the trefoil based only on the formal properties of instanton homology. In fact one can extend such arguments a little further. For example, in the case of the 2-stranded torus knot $K_{2,2 \ell+1}$, the ideal $z_{\mathrm{BN}}^{\natural}\left(K_{2,2 \ell+}\right)$ can be shown to be $J^{\ell}$, generalizing the result for the trefoil $(\ell=1)$. Indeed, the complex that computes $I^{\natural}\left(K_{2,2 \ell+1} ; \Gamma_{\mathrm{BN}}\right)$ can be characterized uniquely up to chain-homotopy equivalence. Similar calculations can be made for the 2-component torus links $K_{2,2 \ell}$, for the twist knots, and for some small pretzel knots.

In all these simple cases, the results which are obtained coincide with the results for Heegaard Floer homology, in the version set up in [3]. In particular, in the notation of [3], the Heegaard Floer complex is a complex of free modules over the ring $\mathbb{F}_{2}[\mathrm{u}, \mathrm{w}]$, and the instanton chain complex is obtained by making the base-change

$$
\mathrm{u} \mapsto L, \quad \mathrm{w} \mapsto P
$$

This coincidence for such simple knots is an inevitable consequence of the formal properties that the two theories share, and it is not clear whether it extends much further. In [3], there is a complete symmetry between the variables $u$ and $w$, a symmetry which is also reflected in formal properties of the closely-related invariant $\Upsilon_{K}(t)$ from [13]. If such a symmetry is present in the instanton theory, then it is not apparent on the surface.

An example where a divergence between the instanton theory and the HeegaardFloer theory might be apparent is the torus knot $K_{3,4}$. Based on preliminary calculations, the authors conjecture that the ideal $z_{\mathrm{BN}}^{\natural}\left(K_{3,4}\right)$ is given by

$$
z_{\mathrm{BN}}^{\natural}\left(K_{3,4}\right)=\left\langle L^{3}, L^{2} P, L P^{2}, P^{3}, Y\right\rangle
$$

where

$$
Y=\left(1+T^{-2}\right) P^{2}+L^{2}
$$

On the other hand, the ideal $\mathbb{A}\left(K_{3,4}\right)$ from [3], based on the calculation of the Heegaard Floer homology of torus knots from [11], is

$$
\begin{aligned}
\mathbb{A}\left(K_{3,4}\right) & =\left\langle\mathrm{u}^{3}, \mathrm{u}^{2} \mathrm{w}, \mathrm{uw}^{2}, \mathrm{w}^{3}, Z\right\rangle \\
& =\left\langle\mathrm{u}^{3}, \mathrm{w}^{3}, Z\right\rangle,
\end{aligned}
$$

where $Z=$ uw. In particular, while uw belongs to the ideal in the HeegaardFloer case, the conjectural calculation implies that $L P$ does not belong to the 
ideal $z_{\mathrm{BN}}^{\natural}\left(K_{3,4}\right)$. The calculation for $K_{3,4}$ can be extended to the other torus knots $K_{3, p}$, and the authors hope to return to these and other questions in a future paper.

\section{References}

[1] A. Alishahi. Unknotting number and Khovanov homology. Pacific f. Math., 301(1):15-29, 2019.

[2] A. Alishahi and N. Dowlin. The Lee spectral sequence, unknotting number, and the knight move conjecture. Topology Appl., 254:29-38, 2019.

[3] A. Alishahi and E. Eftekhary. Knot Floer homology and the unknotting number. Preprint, 2018.

[4] M. F. Atiyah and I. G. Macdonald. Introduction to commutative algebra. AddisonWesley Publishing Co., Reading, Mass.-London-Don Mills, Ont., 1969.

[5] J. Batson. Nonorientable slice genus can be arbitrarily large. Math. Res. Lett., 21(3):423-436, 2014.

[6] P. B. Kronheimer. An obstruction to removing intersection points in immersed surfaces. Topology, 36(4):931-962, 1997.

[7] P. B. Kronheimer and T. S. Mrowka. Knot homology groups from instantons. $\mathcal{F}$. Topol., 4(4):835-918, 2011.

[8] P. B. Kronheimer and T. S. Mrowka. Gauge theory and Rasmussen's invariant. F. Topol., 6(3):659-674, 2013.

[9] P. B. Kronheimer and T. S. Mrowka. Instantons and Bar-Natan homology. Preprint, 2019.

[10] P. Ozsváth and Z. Szabó. Knot Floer homology and the four-ball genus. Geom. Topol., 7:615-639, 2003.

[11] P. Ozsváth and Z. Szabó. On knot Floer homology and lens space surgeries. Topology, 44(6):1281-1300, 2005.

[12] P. S. Ozsváth, A. I. Stipsicz, and Z. Szabó. Grid homology for knots and links, volume 208 of Mathematical Surveys and Monographs. American Mathematical Society, Providence, RI, 2015. 
[13] P. S. Ozsváth, A. I. Stipsicz, and Z. Szabó. Concordance homomorphisms from knot Floer homology. Adv. Math., 315:366-426, 2017.

[14] P. S. Ozsváth, A. I. Stipsicz, and Z. Szabó. Unoriented knot Floer homology and the unoriented four-ball genus. Int. Math. Res. Not. IMRN, (17):5137-5181, 2017.

[15] J. Rasmussen. Khovanov homology and the slice genus. Invent. Math., 182(2):419447, 2010.

[16] C. Scaduto. On definite lattices bounded by a homology 3-sphere and Yang-Mills instanton Floer theory. To appear.

[17] C. W. Scaduto and M. Stoffregen. The mod two cohomology of the moduli space of rank two stable bundles on a surface and skew Schur polynomials. Canad. F. Math., 71(3):683-715, 2019.

[18] I. Zemke. Knot Floer homology obstructs ribbon concordance. Preprint, 2019.

[19] I. Zemke and A. Levine. Khovanov homology and ribbon concordance. Preprint, 2019. 